\title{
INVENTORY RECORD INACCURACY - THE IMPACT OF STRUCTURAL COMPLEXITY AND LEAD TIME VARIABILITY
}

\author{
Salvatore Cannella*; Roberto Dominguez,** Jose M. Framinan* \\ *Industrial Management \& Business Administration Department, School of Engineering, University of \\ Seville, Spain \\ **Centre for Management Studies, Instituto Superior Técnico, Technical University of Lisbon, Portugal
}

\begin{abstract}
This article presents a rigorous prospective analysis of the impact of Inventory Record Inaccuracy (IRI) on complex multi-echelon Supply Chains (SCs). Specifically, key operational factors (i.e., the magnitude of the error, frequency of the inventory audits and lead time variability) and SC structure are systematically assessed. We find that the detrimental effects of IRI are exacerbated by the structural complexity of the SC and lead time variability. Furthermore, we show how the efficacy of countermeasure strategies may vary depending on SC configuration and operational conditions. These results allow us to provide interesting managerial recommendations to guarantee investment in prevention and correction strategies.
\end{abstract}

Keywords: Supply chain management, inventory errors, bullwhip effect, dynamic performance, inventory audits, lead time, multi-agent model, simulation. 


\section{CONTEXT}

Inventory record inaccuracy (IRI) refers to the discrepancy between physical inventory held in stock and the record of inventory stored in a firm's information system (Kok and Shang 2014). This discrepancy can deeply affect the performance of firms (Sarac et al. 2010) by generating lost sales, delay penalties, re-scheduling, suboptimal planning and the increased use of small transport vehicles, among others (Thiel et al. 2010, Cannella et al. 2015). Such inefficiencies are a natural consequence of uncorrected order patterns generated by the Supply Chain (SC) members affected by inventory errors; essentially, they create critical distortions in order placement, as almost every order policy utilizes information regarding current inventory levels (Bruccoleri et al. 2014), and if the recorded inventory quantity does not match the actual quantity on the shelf, the system will either order unnecessary items or fall short on orders (Metzger et al. 2013).

Several studies demonstrate IRI to be a significant problem in practice (Heese 2007, Uckum et al. 2008, Sahin and Dallery 2009, Hardgrave 2013, Kull et al. 2013, Fan et al. 2015). Kang and Gershwin (2005) report an inventory accuracy of only $51 \%$ after a manual inventory verification of 500 stores of a global retailer. DeHoratious and Raman (2008) observe inaccuracies in $65 \%$ of 369.567 inventory records collected from 37 leading retailers in the USA. For example, in 2009, retailers in the USA lost more than $\$ 33$ billion due merely to shrinkage, one of the several root causes of IRI (Hollinger and Adams 2010, Xu et al. 2012). Furthermore, a 10\% profit reduction due to inventory errors has been estimated (Hesse 2007, Hardgrave 2013).

\subsection{Background}

Understanding the impact of this phenomenon on SC performance is important for effective SC management (Mersereau 2013, Ketzenberg et al. 2013). For this reason, there is increasing interest in investigating this issue (Dai and Tseng 2012, Kull et al. 2013). During the last decade, several efforts have been undertaken by researchers and practitioners to understand the causes, consequences and remedies of these detrimental occurrences (see, e.g., Dehoratius et al. 2008, Sahin and Dallery 2009, Rekik et al. 2009, Rekik 2011, Chan et al. 2012, Chen et al. 2014, Sarac et al. 2015). However, there remains substantial room for analyzing and limiting the IRI problem. Among the current gaps in the IRI literature, the following should be taken into account: 
[1] Dynamic effects of Inventory Errors. The literature on IRI may be classified into two streams of research: empirical and theoretical. Empirical research primary investigates the possible correlation between operating conditions and IRI (Hardgrave et al. 2013). Theoretical studies may be further classified into two substreams. The former focuses on the optimization of inventory policies in the presence of errors (Sahin et al. 2009), whereas the latter focuses on the impact of IRI on the behavior of SCs (Cannella et al. 2015). Both theoretical streams are complementary and relevant to understanding and avoiding/reducing IRI occurrences. The majority of IRI studies belong to the first theoretical stream. Such works usually focus on determining the required buffer size to minimize shortage costs by adopting single-period analytical models (Sarac et al. 2010, Thiel et al. 2010) or investigate optimal IRI-preventive and -corrective strategies, such as inventory audits, improved tracking of stored items, etc. (Rekik and Sahin 2012). In contrast, studies that belong to the second theoretical stream usually perform whatif analyses in order to provide scientific and managerial insights on the impact of IRI (i.e., the type and magnitude of inventory error occurrences) and on the effectiveness of countermeasure actions for different market and operational scenarios. In the last decade, prospective analyses have focused on the consequences of and remedies to inventory errors (see, e.g., Fleisch and Tellkamp 2005, Sari 2008, Natchmann et al. 2010, Dai and Tseng 2012), mostly measured in terms of holding costs and customer service. Rarely have investigations shed light on the impact of IRI on critical time-varying phenomena, such as the so-called bullwhip effect (Lee et al. 1997, Hussain et al. 2012, Turrisi et al. 2013, Shan et al. 2014, Li and Zhang, 2015, Zissis et al. 2015, Wang and Disney 2015). However, the consequences of inventory errors include inefficiencies in inventory management and process control at one stage and, by nature, propagate through the SC and may negatively impact the performance of upstream partners (Gel et al. 2010, Dai and Tseng 2012, Xu et al. 2012, Bruccoleri et al. 2014, Kok and Shang 2014, Kwak and Gavirneri 2014, Cannella et al. 2015, Sari 2015).

[2] IRI in multi-echelon SC networks. Most IRI analyses are merely performed for a single-stage SC (Sarac et al. 2010) and, in particular, the retailer stage (Rekik 2011). On the other hand, the few studies investigating the dynamic effects of IRI on multi-echelon SCs focus mostly on classical serial SCs (i.e., SCs with a single 
member in each echelon) (Wan and Evers 2011). The adoption of this multiechelon model represents a common and powerful assumption for analyzing SC performance (Chatfield 2013). However, it has been seldom verified in real SCs (Bhattacharya and Bandyopadhyay 2011, Dominguez et al. 2015a) because the relationships among firms in dynamic SCs are more complicated than those in simple one-to-one buyer-supplier connections (Evers 1999, Wan and Evers 2011). The challenges imposed by the new generation of SCs (see, e.g., Christopher and Holweg 2011, Stank et al. 2011, Christopher and Ryals 2014, Waller and Fawcett 2014, Dominguez et al. 2014a, b, Merzifonluoglu 2015, Annarelli and Nonino 2016, $\mathrm{Li}$ and Zhen 2016) advocate for more realistic models with which to analyze the growing complexity of those structures. Therefore, it would be beneficial to assess the impact of errors in SCs that are characterized by more than one member in the same echelon of the chain, such as divergent SCs (Cannella et al. 2015, Sari 2015). In fact, even though there are different types of SC structures in the real world (e.g., dyadic, serial, convergent, and divergent) (Giard and Sali 2013), the most widely used is the divergent/distribution SC (Beamon and Chen 2001, Hung 2011), which has been adopted in consumer-oriented industries (Hung 2011).

[3] IRI problems and avoiding techniques in real-world SCs: The dynamic performance of SCs depends on both the SC's design (Disney et al. 2004, Chatfield et al. 2012, Dominguez et al. 2015a, Cardoso et al. 2015, Heckmann et al. 2015) and its operational parameters (Wan and Evers 2011, Talluri et al. 2013, Guertler and Spinler 2015). Similarly, IRI, ceteris paribus, may produce a different impact, depending on type of SC and parameter conditions. This consideration can be reasonably extended regarding the efficacy of IRI-limiting strategies, i.e., (1) prevention and (2) correction strategies. Most studies focusing on the application of IRI-limiting strategies usually do not emulate real conditions regarding some key operational factors, most notably, the intrinsic stochasticity of lead time. Because this factor may greatly alter the impact of inventory errors on SC performance and the efficacy of IRI-limiting strategies, it is of interest to investigate this potential effect. 


\subsection{Objective}

Motivated by the previously mentioned considerations, in this paper, we analyze the impact of IRI on the dynamic performance of both simple and complex SC structures under a variety of operational parameter conditions.

More specifically, we model and simulate three different SCs characterized by an increasing level of structural complexity under different IRI scenarios. To emulate the IRI scenarios, we model a gap between the physical inventory, (i.e., the units actually available in stock) and the inventory record (i.e., the units that, according to the information system, are available in stock) of each SC member by introducing into the inventory system a transaction-independent error, more specifically, a shrinkage error (Kull et al. 2013). Among all sources of inventory inaccuracies, this type of error has the biggest impact on SC costs (Fleisch and Tellkamp 2005, Gumrukcu et al. 2008, Uçkun et al. 2008, Zhu et al. 2012, Fan et al. 2014, Kok and Shang 2014, Cannella et al. 2015). The shrinkage error is a permanent inventory loss, resulting in smaller actual inventory when compared to the records in the Information Technology (IT) system (Gel et al. 2010, Dai and Tsang 2012, Sarac et al. 2015). Therefore, it generates an overestimation of the actual stock; the system believes it has inventory on hand (i.e., phantom inventory) and thus fails to order new inventory (Hardgrave 2013).

In order to provide comprehensive data inputs, we adopt a full-factorial experimental design (Evers and Wan 2012). More specifically, the studied factors are (1) the level of inventory errors, (2) the structural complexity of SCs, (3) the lead time variance (as a measure of the lead time variability) and (4) the time between inventory corrections. As a result, we statistically infer the significance of IRI on complex SCs and how the errors interact with other key operational parameters.

We use simulations to perform the analyses. This approach is appropriate because (1) it incorporates a high level of detail regarding the factors of interest, (2) it accommodates nonlinearities essential to IRI research (e.g., frequencies in cycle counting and record corrections), and (3) it accounts for stochastic elements in lead times, inventory errors, and demand across channels (Kull et al. 2013). Due to the nature of our study, we adopt the Multi-Agent System (MAS) modeling approach, recognized as a useful methodology to perform complex prospective SC analysis (Long 2014). In order to 
perform a systematic simulation analysis, we adopt reasonable assumptions and data inputs for simulations obtained from different cases and behaviors to emulate real-world logistic systems (Rabinovic and Cheon 2011). Dynamic performance is measured through the order rate variance ratio, also known as the bullwhip ratio (Chen et al. 2000), a common metric adopted for the evaluation of SC dynamics.

Our study provides novel insights into the IRI phenomenon. More specifically, we show how:

- Detrimental effects of IRI on SC performance are exacerbated by the structural complexity of the SC and lead time variability.

- The resilience of IRI-limiting strategies may be altered by the SC design and operational factors. Ceteris paribus, as structural complexity and lead time variability increase, SCs become more sensitive to the frequency of inventory audits.

These results allow us to provide some managerial considerations to avoid IRI consequences by streamlining the efficacy of IRI-limiting strategies; these considerations include:

(1) The risks related to the adoption of specific policies regarding the frequency of a cycle counting policy are more relevant for complex SCs with high lead time uncertainty.

(2) Investments in IRI-limiting strategies may not be effective due to the complexity of the SC and the lead time uncertainty.

(3) The efficacy of IRI-limiting strategies can be improved by adopting complementary approaches such as limiting lead time variability (i.e., process simplification using lean and six sigma methodologies, improving transportation outsourcing services, collaboration, integration and sharing benefits among partners) and reducing SC complexity (i.e., lean structure design and reengineering SCs to improve IRI-limiting strategies).

The rest of the paper is organized as follows: Section 2 describes the SC models and methodology. Section 3 presents the experimental design and results. Section 4 describes the findings. Section 5 presents directions for SC managers. Finally, Section 6 presents conclusions, limitations and further research directions. 


\section{MODEL DESCRIPTION AND METHODOLOGY}

In this section, we first describe the general design of the simulated SCs. A detailed description of the SC models follows with definitions of the operational aspects. Finally, we provide a description of the MAS SC-simulation tool (SCOPE) used to model and simulate the SCs. A summary of the nomenclature used in this work is provided in Table 1.

\begin{tabular}{|c|c|c|c|}
\hline$E$ & Total number of echelons & $s_{L_{i}^{i}}^{2}$ & Estimated variance of the lead time of $n_{l}$ \\
\hline$N$ & Total number of nodes & $\rho$ & $\begin{array}{l}\text { Inventory review period of Order-Up-To (OUT) } \\
\text { policy }\end{array}$ \\
\hline $\operatorname{Div} F$ & Divergence factor & $S_{L}^{t}$ & Desired level of stock of $n_{L}$ \\
\hline$I$ & Echelon position in the SC & $z$ & Safety factor for the OUT policy \\
\hline$j$ & Node position in echelon $i$ & l & Moving averages and variance forecast period \\
\hline$u_{l}$ & Node at position $j$ in echelon $i$ & $P_{t}^{t}$ & Physical inventory of $u_{l}$. \\
\hline$N_{l}$ & Total number of nodes in echelon $i$ & $K t$ & Recorded inventory of $n_{l}$ \\
\hline$N_{\mathrm{L}}$ & Total number of customers & Shr & Shrinkage factor \\
\hline$C_{j}$ & Customer at position $j$ & $\Delta t_{l}^{t}$ & Inventory lost by $n_{l}$ due to shrinkage \\
\hline$T$ & Current simulation time & $T B C$ & Time between inventory corrections \\
\hline$V_{L}^{t}$ & Demand placed by $C_{J}$ & $O_{t}^{t}$ & Orders placed by $n_{l}$ \\
\hline$\mu_{D_{E}}$ & Average demand placed by $C_{l}$ & $N t_{i}^{t}$ & Net stock of $n_{l}$. \\
\hline $\bar{D}_{\mathrm{L}}^{t}$ & Estimated average demand placed by $C_{J}$ & $W \stackrel{t}{l}$ & Work in progress of $n_{L}$ \\
\hline$a_{D_{C}}^{z}$ & Variance of demand placed by $C_{j}$ & $B_{l}^{t}$ & Backlog of $n_{l}$ \\
\hline$s_{D_{L}^{L}}^{2}$ & Estimated variance demand placed by $C_{\mu}$ & $T$ & Total Variance Amplification echelon $i$ \\
\hline$D_{i}^{t}$ & Demand faced by $n_{L}$ & $T$ & Total simulation time (excluding warm-up) \\
\hline $\bar{D}_{L}^{t}$ & Estimated average demand faced by $n_{l}$ & $s_{U}^{2}$ & Estimated variance of orders placed by echelon $i$ \\
\hline$s_{D_{l}^{l}}^{z}$ & Estimated variance demand faced by $n_{L}$ & $a_{U_{1}}^{2}$ & Variance of orders placed by $n_{l}$ \\
\hline$L_{l}^{t}$ & Lead time of $u_{l}$ & $s_{U_{1}^{L}}^{2}$ & Estimated variance of orders placed by $n_{l}$ \\
\hline$\mu_{L_{I}}$ & Average lead time of $n_{l}$ & $B w S l$ & Bullwhip slope \\
\hline $\bar{L}_{l}^{t}$ & Estimated average lead time of $n_{l}$ & $\pi_{l}$ & Position of the ith echelon \\
\hline$a_{L_{l}}^{2}$ & Variance of the lead time of $n_{l}$ & SC_Complx & Supply chain complexity \\
\hline
\end{tabular}

Table 1. Nomenclature. 


\subsection{Configuration of the supply chains}

In the SC dynamics literature, the "gold standard" SC model is the four-echelon serial SC, i.e., 1 Factory, 1 Distributor, 1 Wholesaler and 1 Retailer (see, e.g., Sterman 1989, Chatfield et al. 2004, Dejonckheere et al. 2004, Cannella and Ciancimino 2010, Garcia et al. 2012, Chatfield 2013, Croson et al. 2014, among others). The results obtained by adopting this model have been validated in the real business world and have contributed considerably to advances in the related scientific knowledge.

However, it has recently been shown that serial SCs and more structurally complex SCs, ceteris paribus, perform differently (Sodhi and Tang 2011, Dominguez et al. 2014a). Thus, to enhance the analysis of the impact of IRI on multi-echelon SCs, we analyze this noxious phenomenon on not only a serial SC but also on other possible configurations characterized by an increasing level of structural complexity. To do so, we simulate IRI in a number of exemplificative SC configurations representing increasing levels of structural complexity.

In order to select appropriate SC configurations, we refer to three well-known factors identified in the literature as the drivers of SC structural complexity (see, e.g., Sucky 2009, Dominguez et al. 2015a): (1) the total number of echelons ( $E$ ) or functional levels of the chain; (2) the total number of nodes $(N)$, which indirectly accounts for the average number of nodes per echelon; and (3) the divergence factor (DivF), which provides information on how the nodes are distributed along the different echelons of the chain. Assuming a SC with $i=1 \ldots E$ echelons and $j=1 \ldots N_{l}$ nodes in each echelon $i$, the divergence factor is calculated as the standard deviation of the number of nodes across the echelons of the SC $\left(N_{l}\right)$ related to the average number of nodes in each echelon (N/E), as in Eq. (1) (Dominguez et al., 2015a).

$$
D=\sqrt{\sum_{l=1}^{E} \frac{\left(N_{i}-N / E\right)^{2}}{E-1}}
$$

The first configuration is the previously mentioned serial four-echelon configuration, termed in the following the Low Complexity SC (LCSC). We adopt this model to emulate the configuration with a low level of structural complexity $(E=4, N=4$, $\operatorname{DivF}=0)$. 
The second and third configurations are two divergent/distribution SCs, i.e., the Medium Complexity SC (MCSC) and the High Complexity SC (HCSC). These SCs emulate medium and high levels of structural complexity, respectively. As ncombinations of divergent SCs can be generated, to establish a consistent design of these structures and to represent increasing levels of SC structural complexity, we adopt the following criteria:

- To provide a benchmark for the different SC topologies and to isolate the main effects, the divergent SCs have to be analogous to the LCSC, which is used as a reference. Thus, both divergent SCs should have an identical number of echelons, i.e., $E=4$.

- Because a divergent distribution is characterized by a tree-like structure in which every stock point in the system receives supply from a stock point exactly one level higher and supplies to one or more lower-level stock points (Hwarng et al. $2005)$ and there is a fixed number of echelons $(E=4)$, it is necessary to modify the number of facilities at each echelon and the number of links between the locations. Hence, to generate SCs characterized by higher structural complexity, both the MCSC and the HCSC have to be characterized by a higher $N$ and DivF than the LCSC.

Thus, the MCSC is constructed by allowing each node to supply two nodes downstream, thus obtaining a divergent SC with 15 nodes distributed over 4 echelons $(\mathrm{E}=4, \mathrm{~N}=15, \mathrm{DivF}=3.1)$ as follows: 1 Factory, 2 Distributors, 4 Wholesalers and 8 Retailers. This structure has been previously adopted in the SC dynamic literature (see, e.g., Dominguez et al. 2014a, Dominguez et al. 2015b), and it has been shown to adequately represent increasing structural complexity with respect to the classic LCSC.

The HCSC, which is generated by adopting an analogous procedure as that for the MCSC, is the SC with the highest $N$ and $\operatorname{DivF}(E=4, N=22, D i v F=4.8): 1$ Factory, 3 Distributors, 6 Wholesalers and 12 Retailers (Figure 1). 


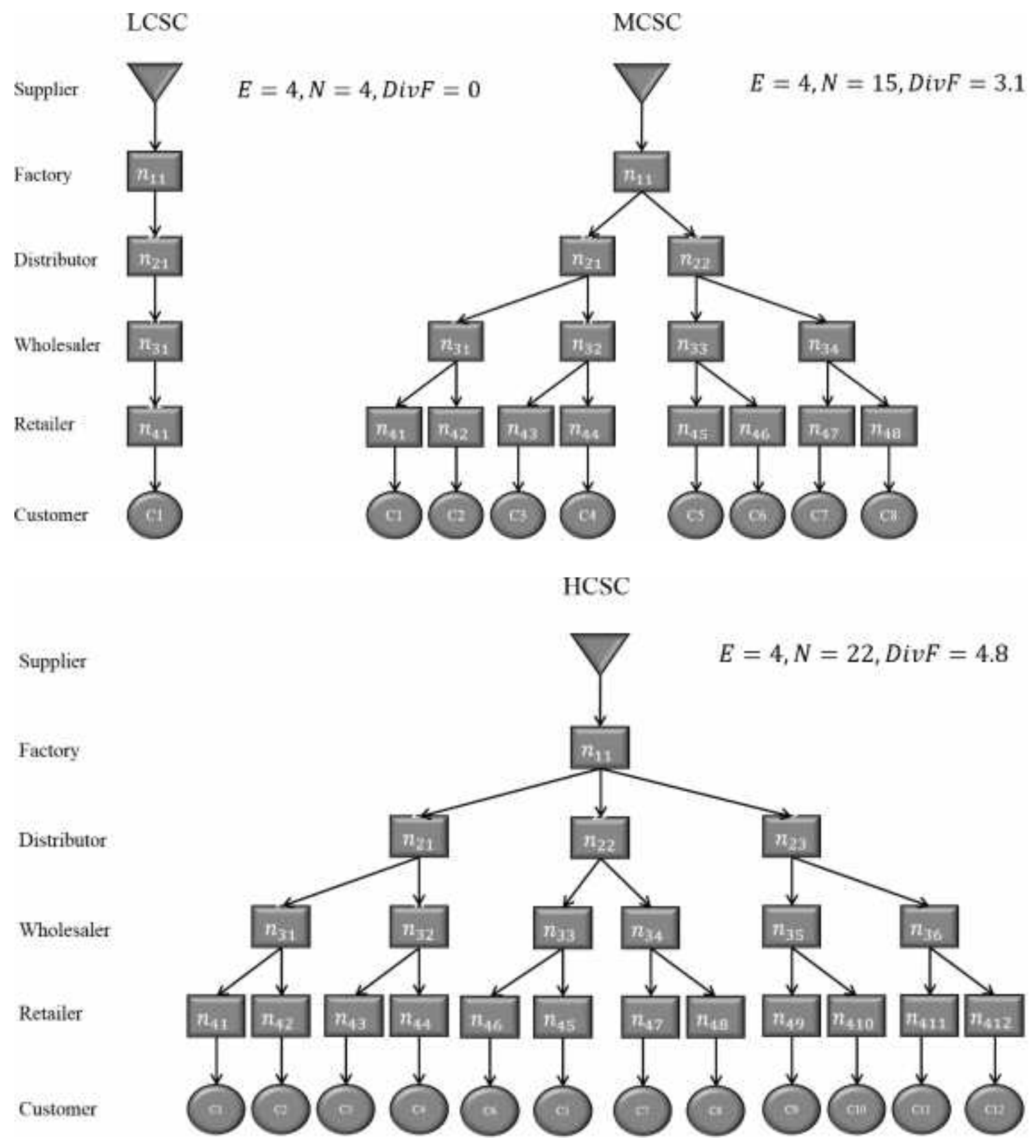

Figure 1. SCs under analysis.

\subsection{Supply chain model and assumptions}

The SC model relies on widely accepted assumptions in SC dynamics and IRI studies. Among these are the following:

- Stochastic demand placed by customers. The demand of each customer $j$ follows a normal distribution with mean $\mu_{D_{C}}$, estimated by $\bar{D}_{L}^{t}$, and variance $\sigma_{D_{L}}^{2}$, estimated by $s_{D_{C}^{E}}^{2}$. Customers do not fill orders. Customer demands are mutually independent. 
- Factories place orders to an outside supplier with unlimited capacity.

- The lead time $\left(L_{l}^{t}\right)$ of node $j$ in echelon $i$ is assumed to be stochastic and gamma distributed (Chatfield et al., 2004). Lead times are assumed to be stationary, independent and identically distributed with mean $\mu_{L_{l}}$ estimated by $\bar{L}_{l}^{t}$ and variance $o_{L_{l}}^{2}$ estimated by $s_{L_{l}^{t}}^{2}$.

- The adopted inventory policy is the $(\rho, S)$ or periodic review with the Order-UpTo (OUT) level (Nachtmann et al. 2010, Chatfield and Pritchard 2013, Li et al 2014). Given the common practice in retailing to replenish inventories frequently (Disney and Lambrecht 2008), OUT is the most largely used periodic review policy in practical applications (Hax and Candea 1984). The OUT level, $S_{l}^{t}$, is the base stock that allows the system to meet the demand during the time period $L_{l}^{t}+\rho$, which is also known as the "protection period" (Chatfield et al. 2004). Under this policy, orders are placed at discrete time intervals given by $\rho$, the inventory review interval. Prior to placing an order, the system checks the inventory position (explained below, (Eq. (3)) and determines if it is below the OUT level $S_{l}^{t}$. In this case, an order is placed for the difference between the OUT level and the inventory position; otherwise, no order is placed. Thus, negative orders (returns) are not permitted (see below). The OUT level is calculated with a commonly used approximation (Eq. (2)), where $z$ is a safety factor:

$$
S_{l}^{t}=\left(\bar{L}_{l}^{t}+\rho\right) \bar{D}_{l}^{t}+z \sqrt{\left(\bar{L}_{l}^{t}+\rho\right) s_{D_{l}^{t}}^{2}+\bar{D}_{l}^{t^{2}} s_{L_{l}^{t}}^{2}}
$$

- In order to update the $S_{l}^{t}$, node $j$ in echelon $i$ needs to forecast the incoming demand $\left(\bar{D}_{l}^{t}\right.$, the expected average demand at time $t$, and its variance, $\left.s_{D_{l}^{E}}^{2}\right)$ and the lead time of upstream partners $\left(\bar{L}_{l}^{t}\right.$, the expected average lead time at time period $t$ and its variance, $s_{L_{l}^{\leftarrow}}^{2}$ ). Demand and lead time data from previous periods are available at each node. To estimate $\left(\bar{D}_{l}^{t}, s_{D_{l}^{E}}^{2}\right)$, each node uses a $t$-period moving average and a $t$-period moving variance. To estimate $\left(\bar{L}_{l}^{t}, s_{L_{l}^{\tau}}^{2}\right)$, each node uses running averages. 
- In order to simulate the IRI phenomenon, we considered two types of inventories: the physical inventory, i.e., the physically available units $\left(P_{l}^{t}\right)$, and the recorded inventory $\left(K_{l}^{t}\right)$, i.e., the inventory available according to the information system. The discrepancy between the physical inventory level and inventory records is due to IRI.

- According to Kull et al. (2013), inventory errors may be classified into two main groups: (1) transaction-dependent errors and (2) transaction-independent errors (Lee and Ozer 2007; Nachtmann et al. 2010). The former are changes in IRI triggered by replenishments, demand orders, or product returns (e.g., incorrect deliveries, misplaced items or incorrect picking). The latter are changes in IRI that occur irrespective of transactions and that are influenced by the amount of on-hand inventory (e.g., shrinkage, spoilage, or damage) (Kull et al. 2013). In this work, IRI is assumed to be caused by transaction-independent errors due to shrinkage, and it is assumed to be present at each node of the SC. We focus merely on shrinkage for three reasons: (I) as claimed by Gel et al. (2010), analyses of inventory errors due to different causes need to be studied separately because the actions taken to address the different causes may be quite different; (II) as reported in the literature, shrinkage has the largest impact on SC costs (Cannella et al. 2015 and the references therein); and (III) despite the impact and the presence of shrinkage errors in practice, the literature focusing on shrinkage errors is particularly limited (Rekik and Sahin 2012). According to Khader et al. (2014), shrinkage can be modeled by a multiplicative error structure because the error magnitude might depend on the quantity of stock. Thus, the inventory lost by each node at time period $t$ can be modeled as $I{ }_{l}^{t}=S h r \cdot P_{l}^{t}$, where $S h r$ is the shrinkage factor. This factor considers random values every time period according to a predefined interval.

- Each member of the SC adopts a periodic inventory cycle counting policy. Therefore, recorded inventory is updated to the real level of physical inventory at periodic intervals. This span is defined as the time between corrections (TBC), (Nachtmann et al. 2010), also known in the literature as inventory alignment time (Fleisch and Tellkamp 2005, Cannella et al. 2015). Both the physical inventory and the inventory records are reconciled by updating the recorded inventory to the actual physical inventory. Clearly, a $T B C$ of one 
period is equivalent to no inventory errors because the inventory records are reconciled every period.

- While most analytical models assume that the entire SC is able to backorder, real-world systems rarely include backordering at the retail store level (Nachtmann et al. 2010). Therefore, in the event of a stock-out situation at the retailer's echelon, backordering is not allowed, and unfilled demand is lost. On the contrary, companies in higher echelons are allowed to backorder.

- We assume that returns of excess inventory to upstream partners are not permitted, mainly because the allowance of returns, although a common assumption in the bullwhip effect literature, may not be realistic and may overestimate the bullwhip effect (Chatfield and Pritchard 2013, Dominguez et al. 2015b).

- Orders $\left(O_{l}^{t}\right)$ are placed by each node at each time period in order to raise their current inventory position to the target inventory level $S_{l}^{t}$. The inventory position equals the net stock $\left(\begin{array}{ll}t & t \\ l\end{array}\right)$ plus the inventory on order but not yet arrived, or work in progress ( $\left.\begin{array}{ll}W & t \\ l\end{array}\right)$ (Disney and Lambrecth 2008). The net stock equals recorded inventory minus backlog $\left(B_{l}^{t}\right)$, with the exception of retailers, which are not allowed to backorder (Eq. (3)). Notice that due to the presence of inventory errors, orders are placed according to erroneous information about the current inventory $\left(K_{l}^{t}\right)$.

$$
O_{l}^{t}=S_{l}^{t}-K_{l}^{t}+B_{l}^{t}-W \quad{ }_{l}^{t} ; O_{l}^{t} \geq 0
$$

- In each time period, each node performs the following sequence of actions from downstream echelons to upstream echelons:

1. Update the OUT level $\left(S_{l}^{t}\right)$ using the forecast calculated in the previous period.

2. Place an order $O_{l}^{t}$ to raise or lower the inventory position to $S_{l}^{t}$.

3. Receive products from the upstream partner and update $\left[P l_{l}^{t}, K_{l}^{t}\right]$ accordingly.

4. Receive new orders from downstream nodes, satisfy demand and update $\left[P{ }_{l}^{t}, R_{l}^{t}\right]$ accordingly. 
5. Calculate a new forecast to be used in the next period.

6. Lose inventory due to shrinkage $I{ }_{l}^{t}$, updating $P_{l}^{t}$ accordingly.

7. Perform inventory cycle counting and update the recorded inventory in their information systems $K_{l}^{t}=P_{l}^{t}$ at every TBC interval.

\subsection{Methodology: a MAS-based supply chain simulator (SCOPE)}

An SC is a complex adaptive system (Surana et al. 2005, Pathak et al. 2007, Chen2012) that often shows complex structures, uncertainties and partial information (Long and Zhang 2014). The numerous interactions among entities as well as the characteristics of nonlinearity, dynamics, and uncertainty, among others, make it challenging to analyze SCs and predict their responses over time ( $\mathrm{Li}$ et al. 2010a, b). Traditional analytical methods are thus ineffective in SC modeling (Long et al. 2011) because they rely on mathematical formalizations of the SC and thus require simplified approximations, which are usually restrictive and limited in their consideration of time (Long and Zhang 2014).

To overcome the inconveniences and limitations of these analytic methods, simulations have been broadly used to model and evaluate a wide range of different strategies in SC management and have served as a decision-making tool to improve SC performance, as simulations are able to handle the dynamics and stochastic behavior of the inter-related SC processes (Chan and Prakash 2012) with strong and realistic modeling and analysis capabilities. In addition, simulations have great ease of use, which makes this methodology increasingly popular (Evers and Wan 2012). MAS is a modeling and simulation paradigm from the field of artificial intelligence that provides the best mechanism for modeling the autonomy, communication, coordination, and decision making of an SC (Long and Zhang 2014). In addition, there is a natural correspondence between SC participants and agents in a simulation model. Therefore, MAS is one of the most effective tools for SC modeling (see, e.g., Swaminathan et al. 1998, Chatfield et al. 2001, Govindu and Chinnam 2010, Chatfield et al. 2012, Dominguez and Framinan 2013, Long and Zhang 2014, among others).

The SCs analyzed in this paper have been modeled by SCOPE (Dominguez and Framinan 2013), which is a MAS-based software platform specifically designed to overcome the shortcomings of traditional methodologies in SC modeling, allowing the 
simulation of large SCs with complex configurations and uncertainties, which are more similar to real systems. The use of random variables allows SCOPE to simulate stochasticity in any business process. In addition, the scalability of the MAS models permits the user to create SCs of any size and distribution. These and other characteristics (please see Dominguez and Framinan 2013 for more information) make SCOPE a good choice for modeling the IRI problem in complex SC configurations with uncertainties.

The simulator was implemented using Java-Swarm, a well-known software platform for agent-based system development. SCOPE has been validated by contrasting the results obtained from the simulations that have been carried out on an SC previously modeled by other authors, such as Chen et al. (2000), Dejonckheere et al. (2004) and Chatfield et al. (2004). For further information on the validation process, please see Dominguez and Framinan (2013).

\section{EXPERIMENTS}

In this section, we first describe the experimental design by defining the variables to be analyzed (independent variables), the experimental outcomes (dependent variables), and the parameters of the model and simulation conditions. Then, we present a statistical analysis of the output data using ANOVA, which analyzes the significance of the independent variables related to the impact on the dependent variables.

\subsection{Experimental design}

A factorial experimental design is generally employed to generate input datasets because it provides comprehensive data input (Evers and Wan, 2012). We adopt a full factorial set of experiments to test the statistical significance of the impact of the experimental factors i.e. shrinkage factor, time between corrections, complexity of the $\mathrm{SC}$, and lead time variance (as a measure of the lead time variability) on the bullwhip effect.

We select three levels for each experimental factor (low, medium, and high), as indicated in Table 2. Therefore, we perform a total of $3^{4}=81$ experiments. In order to select appropriate values for the three levels of the experimental factors, we searched for similar values in relevant studies on this topic. The levels of the shrinkage factor and the 
time between corrections are set according to Nachtmann et al. (2010). Those authors investigate a single-echelon system that is affected by demand error and inventory error, they use a full-factorial design to analyze system performance, and they establish three values of inventory errors and time between corrections. According to the authors, both the model and the chosen values are consistent with real world systems. Thus, we can argue that this setting may better approximate the effect of IRI in real SCs. The value of the shrinkage factor changes every time period according to a uniform distribution within a predetermined interval, which depends on the level of the factor. The time between corrections may vary from 1 period (it is equivalent to a scenario without inventory errors) to 4 and 7 periods. The values chosen for the three levels of lead time variance are typical values from the SC and bullwhip effect literature (see, e.g., Chatfield et al. 2004). For simplicity, we assume that for a given experimental point (a predefined combination of the levels of the experimental factors), the levels of the aforementioned factors are the same for each node of the SC. Finally, the three levels of SC complexity are the three SCs described in Section 2.1 with increasing structural complexity.

The values of the model parameters are common values used in bullwhip effect literature (see, e.g., Chatfield and Pritchard 2013). These values are summarized in Table 2.

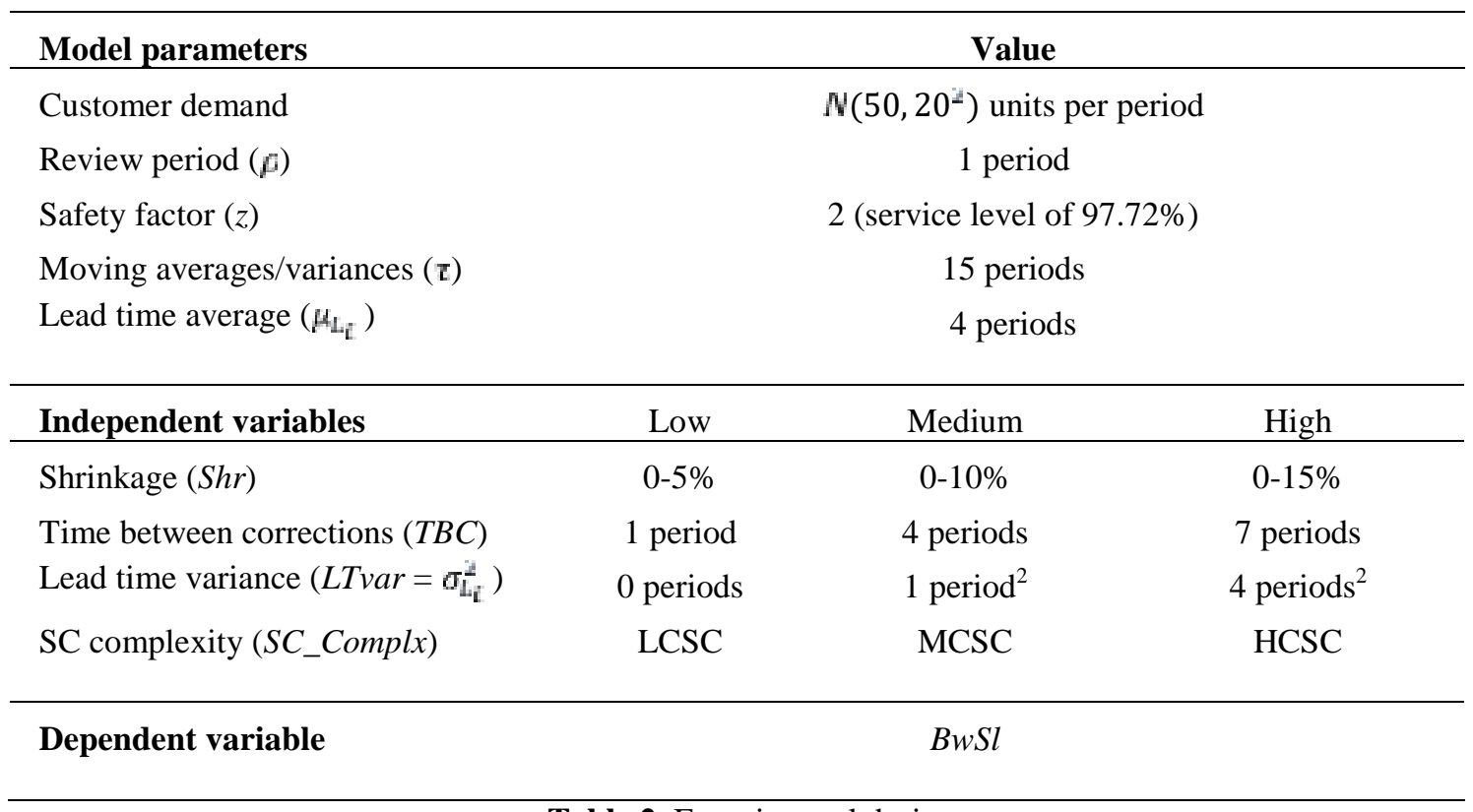

Table 2. Experimental design.

The response variable of the experimental design is the bullwhip slope $(B w S l)$, which is explained below. The bullwhip effect has been traditionally quantified using the Order 
Rate Variance Ratio or Total Variance Amplification (TVAmp) (Chen et al., 2000, Dejonckheere et al. 2004, Chatfield and Pritchard 2013, Chatfield 2013, Cannella 2014), computed as the ratio of the estimated variance of orders placed by the echelon $i$ to the estimated variance of orders placed by customers $s_{U_{t}^{T}}^{2} / s_{D_{C}^{T}}^{2}$. All metrics are computed at the end of the simulation time $(T)$.

In order to apply this metric to divergent SCs for which there might be more than one node in each echelon, we use aggregate measures for each echelon (Dominguez et al. 2015b). Therefore, assuming that all customers' demands are independent and that each node places orders independently, the aggregate variance in echelon $i$ is calculated as the sum of the variances of orders of each node $j$ in that echelon $i\left(\sigma_{U_{l}}^{2}, \sigma_{D_{C}}^{2}\right)$, which are estimated by $\left(\mathrm{s}_{U_{t}^{T}}^{2}, \mathrm{~s}_{D_{L}^{T}}^{2}\right)$. Thus, the calculation of $T \quad$ l for a divergent $\mathrm{SC}$ can be written as in Eq. (4).

$$
T \quad \imath=\frac{\sum_{j=1}^{N_{i}} s_{U_{l}^{T}}^{2}}{\sum_{j=1}^{N_{L}} s_{D_{L}^{T}}^{2}}
$$

In this paper, we are not particularly interested in analyzing the performance of each specific echelon of the SC because it would lead to an elevated number of outputs due to the high number of experiments (see below) and may confuse the interpretation of the results. Instead, we assessed the global performance of the SC, which can be achieved using a global measure of the bullwhip effect that summarizes all of the ratios obtained for each echelon into a single value, the bullwhip slope (BwSl) (Cannella et al. 2013, Dominguez et al. 2015b). This metric is computed as follows. For each echelon, the values of $T \quad$ are plotted in a Cartesian diagram using the echelon position as the independent variable. This interpolated curve is referred to in the literature as the Dejonckheere et al. (2004) curve. The $B w S l$ is the tangent of the angle of inclination of the linear regression of the Dejonckheere et al. (2004) curve. Similarly, the $B w S l$ can be defined as the slope of the linear interpolation of the set of $T \quad p_{l}$ values for a given SC (Eq. (5)). The use of this metric allows for a concise comparison between different SCs at the network level, as it measures the magnitude of the bullwhip propagation. A high value of the slope indicates a fast propagation of the bullwhip effect through the $\mathrm{SC}$, whereas a low value indicates smooth propagation. 


$$
B=t_{l} \quad T=\frac{E \sum_{l=1}^{E} n_{l} T \quad l_{l}-\sum_{l=1}^{E} n_{l} \sum_{l=1}^{E} T}{E \sum_{l=1}^{E} u_{l}^{2}-\left(\sum_{l=1}^{E} n_{l}\right)^{2}}
$$

$\pi_{l}$ is the position of the $i$ th echelon

In order to account for randomness, multiple replications of the experiments are performed, and the simulation outputs are statistically analyzed. According to Evers and Wan (2012), two main factors must be accounted for in order to design the simulation runs: the number of replications and the length of each replication. Regarding the former, more replications lead to greater precision in terms of statistical inferences. Therefore, we perform 30 replications for each set of inputs, obtaining a total of 2,430 (30x81) simulation runs. With respect to the latter, because more-complex SCs need more time to reach a steady state, we use a long simulation time of 4,000 periods with 1,000 warm-up periods up to reduce initialization bias, resulting in an effective simulation time of $T=3,000$ periods. Relevant data regarding the amplification of demand variability are collected for each node of the SCs under study and averaged for the different echelons. Then, $B w S l$ is calculated for each experiment.

\subsection{Analysis of variances (ANOVA)}

In this section, we present the results of an ANOVA on the simulation data, aiming to determine the significance of the experimental factors (independent variables) using the $B w S l$ as the response variable and formally check for possible interactions. Table 3 reports the results. Because high-order interactions are often minimal (Hinkelmann and Kempthorne 1994), only information on the main effects and two-order interactions is presented.

The results show a very good fit, with $R^{2}=99.7 \%$. All the experimental factors are found to be statistically significant $(p<0.001)$ at the $95 \%$ confidence level. The results suggest that all the analyzed factors significantly impact the bullwhip effect. Moreover, all the two-factor interactions are significant $(p<0.001)$ at the $95 \%$ confidence level. 


\begin{tabular}{llllll}
\hline Source & DF & SS & MS & $F$ & $p$ \\
\hline Adjusted Model & 80 & $93,725.074$ & $1,171.563$ & $7,655.832$ & $<0.001$ \\
Shr & 2 & $4,107.362$ & $2,053.681$ & $13,420.219$ & $<0.001$ \\
SC_Complx & 2 & $28,894.113$ & $14,447.057$ & $94,407.382$ & $<0.001$ \\
LTvar & 2 & $21,791.153$ & $10,895.576$ & $71,199.474$ & $<0.001$ \\
TBC & 2 & $14,801.856$ & $7,400.928$ & $48,362.946$ & $<0.001$ \\
Shr $*$ SC_Complx & 4 & $2,099.406$ & 524.852 & $3,429.755$ & $<0.001$ \\
Shr ${ }^{\text {LTVar }}$ & 4 & 635.139 & 158.785 & $1,037.613$ & $<0.001$ \\
Shr $*$ TBC & 4 & $4,820.864$ & $1,205.216$ & $7,875.742$ & $<0.001$ \\
SC_Compl $*$ LTvar & 4 & $5,257.739$ & $1,314.435$ & $8,589.456$ & $<0.001$ \\
SC_Compl $*$ TBC & 4 & $5,469.617$ & $1,367.404$ & $8,935.595$ & $<0.001$ \\
LTvar*TBC & 4 & $2,135.387$ & 533.847 & $3,488.536$ & $<0.001$ \\
Error & 1,539 & 235.511 & 0.153 & & \\
Total & 1,620 & $328,367.335$ & & & \\
\hline
\end{tabular}

Table 3. ANOVA.

\section{DISCUSSION}

In this section, we discuss the results obtained by the ANOVA by first analyzing the main effects of each experimental factor (i.e., analyzing the impact of shrinkage, time between corrections, SC complexity and lead time variability on the response variable when considered independently) and then describing the interactions (i.e., how the mentioned factors impact the response variable when other factors are considered at the same time).

\subsection{Main effects}

We begin the discussion by performing a classification of the experimental factors according to their impact on the bullwhip effect. Adopting the parameter $F$, we observe that SC complexity reveals the highest impact on the bullwhip effect $(F=94,407.382)$, followed by the lead time variability $(F=71,199.474)$, the time between corrections $(F=48,362.946)$ and the inventory shrinkage $(F=13,420.219)$.

In order to describe the main effects of the analyzed factors, we plot the averages of all the experiments for each level of the analyzed factor together with the $95 \%$ confidence intervals in Figure 2. The impact of SC complexity and lead time variability on the bullwhip effect has been previously reported by other authors (see, e.g., Chatfield et al. 2004, Dominguez et al. 2015a). Therefore, we do not describe the main effects of these 
factors in detail. Instead, we report an increasing bullwhip effect as the SC complexity or the lead time variability increase (Figure 2), which is in line with the aforementioned studies.

The plot situated at the upper left of Figure 2 reveals a progressive intensification of the bullwhip effect as the level of inventory shrinkage increases. This indicates that a minimum increase of inventory shrinkage inevitably leads to a deterioration of the dynamic performance of the entire SC. Moreover, this detrimental occurrence is observed for all simulated scenarios. Analogously, examining the plot situated at the lower left of Figure 2, we observe that the time between corrections significantly impacts the bullwhip effect. Thus, a decision on the configuration of the inventory cycle counting policy might be relevant to control the bullwhip effect in SCs with IRI. Furthermore, we note a particular phenomenon regarding this factor: we report a low impact on the bullwhip effect for low to medium levels of time between corrections but a substantially higher impact on the bullwhip effect for medium to high levels of this factor. The first finding of our work can be summarized as follows:

- Both inventory errors and the configuration of the inventory cycle counting policy have a significant impact on SC behavior.

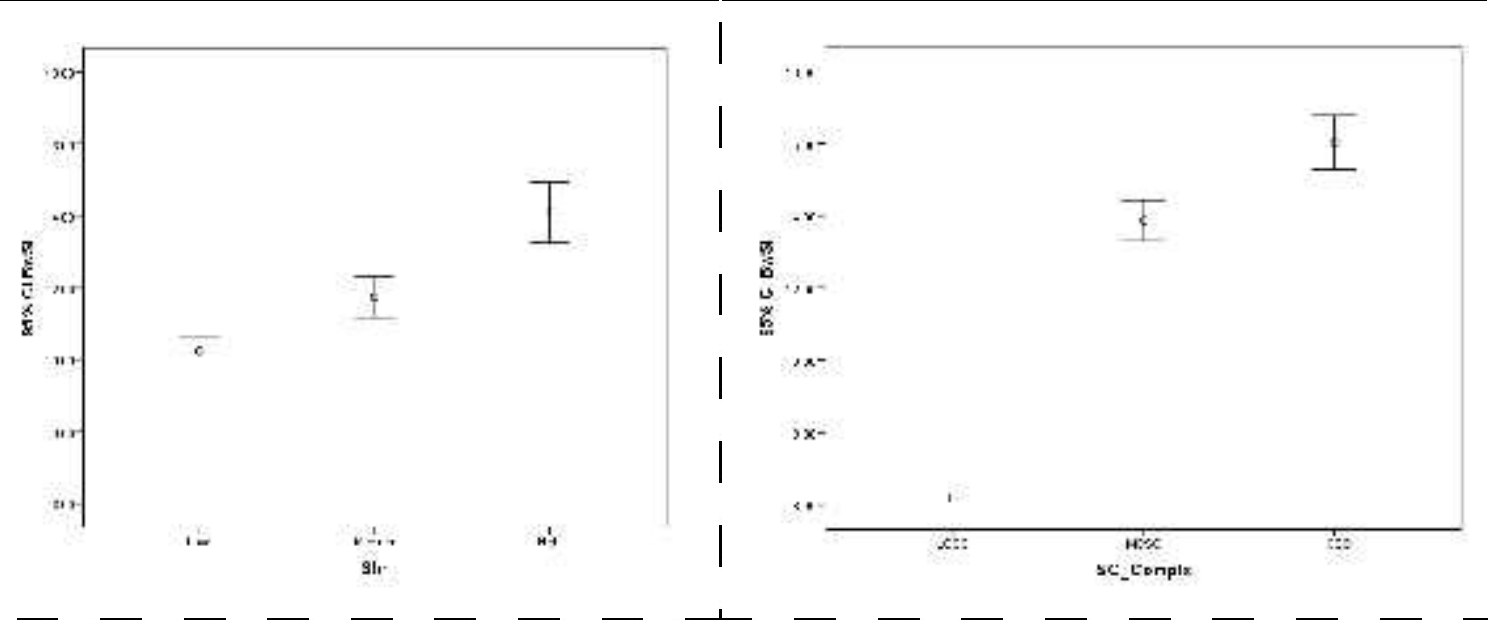




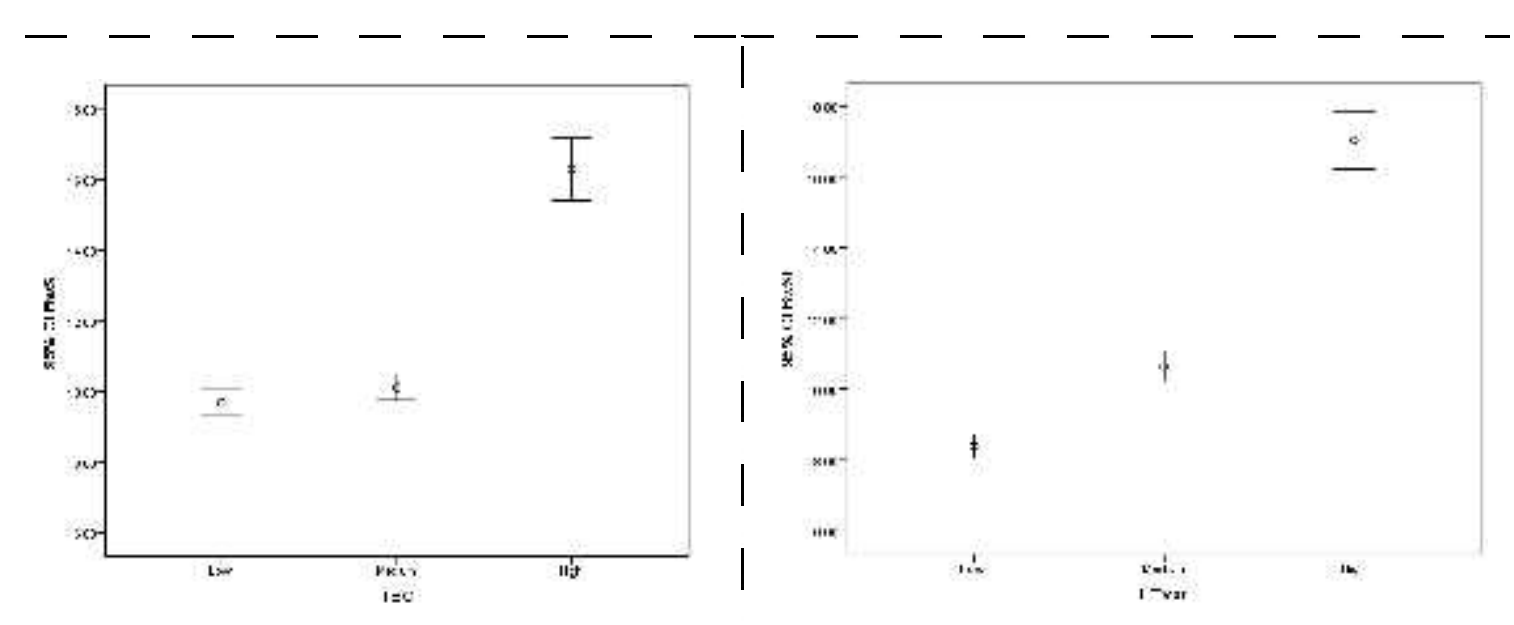

Figure 2. $B w S l$ averages and $95 \%$ confidence intervals for each level of the experimental factors.

\subsection{Interactions}

The previous analysis provides an overview of how the factors under study impact the bullwhip effect. However, due to the significant interactions detected by ANOVA, these factors must be analyzed together. Essentially, the relevance of the inventory errors and the configuration of the inventory cycle counting policy (time between corrections) may depend on the structural complexity of the SC and on lead time variability. Therefore, in the following analysis, we provide a detailed description of the identified interactions.

We first discuss the interaction between inventory shrinkage and the other factors. Then, we describe the interactions related to the time between corrections. Figures 3 to 5 show the interaction plots. Each plot shows the average $B w S l$ for each combination of levels of the interacting factors.

\subsubsection{Inventory shrinkage and supply chain complexity}

The left side of figure 3 reveals an important interaction between inventory shrinkage and SC complexity (notice how the magnitude of the slopes of the Shr-BwSl curves increase at higher levels of $S C_{-}$complx). We estimate that an increase in the shrinkage level from $0-5 \%$ to $0-15 \%$ in the LCSC results in a $B w S l$ increase of $17.07 \%$, whereas the same increase in the shrinkage level in the HCSC results in a $B w S l$ increase of $53.83 \%$. This output provides an interesting and novel finding. SCs affected by the IRI problem and characterized by higher structural complexity suffer a deeper decrease in dynamic performance when compared with SCs of lower structural complexity. Thus, it is reasonable to assume that complex SCs will experience higher benefits in dynamic 
performance by adopting IRI-avoiding policies. We can summarize this finding as follows:

- As the complexity of the SC increases, the impact of inventory errors on performance increases. Therefore, complex SCs are more vulnerable to the IRI problem.

\subsubsection{Inventory shrinkage and lead time variability}

This interaction is shown on the right side of Figure 3. SCs with higher lead time variability are more sensitive to inventory shrinkage. We estimate that an increase in inventory shrinkage from $0-5 \%$ to $0-15 \%$ results in a $~ 34 \%$ increase of $B w S l$ in cases of low or medium levels of lead time variability. However, an analogous inventory shrinkage increase with a high level of lead time variability generates a $42.26 \%$ increase in $B w S l$. This result provides the following finding:

- The impact of inventory errors on performance depends on the lead time variability of the SC. More specifically, SCs facing higher lead time uncertainty are more vulnerable to inventory errors.

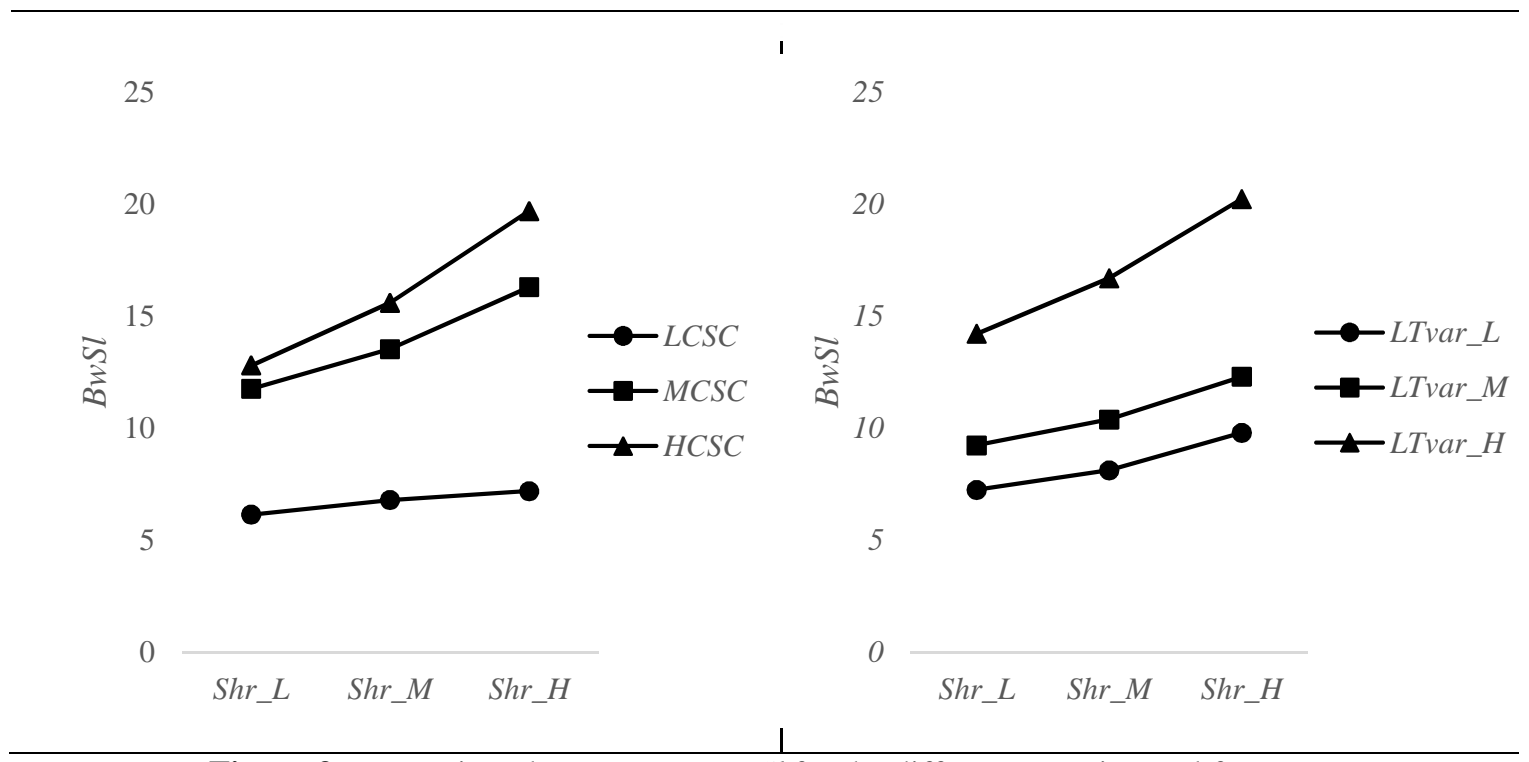

Figure 3. Interaction plots: average $B w S l$ for the different experimental factors.

\subsubsection{Inventory shrinkage and time between corrections}

A relevant interaction is identified between the inventory shrinkage and the time between corrections (Figure 4, left). In fact, for low and medium levels of time between corrections, the impact of the inventory shrinkage on the $B w S l$ is very low. It can be 
estimated that an increase in the inventory shrinkage from $0-5 \%$ to $0-15 \%$ results in a $B w S l$ increase of (1) $7 \%$ for 1 period between corrections and (2) $11.8 \%$ for 4 periods between corrections. In contrast, the impact of the inventory shrinkage on the $B w S l$ is more relevant for high levels of the time between corrections. An increase in the inventory shrinkage from $0-5 \%$ to $0-15 \%$ results in a $B w S l$ increase of $84.6 \%$ for 7 periods between corrections. The analysis of this interaction provides the following finding:

- The impact of inventory errors on performance significantly depends on the value of the time between corrections. More specifically, as this value increases, the SC becomes more vulnerable to inventory errors.

\subsubsection{Time between corrections and supply chain complexity}

The most significant interaction $(F=8,935.595)$ takes place between the time between corrections and the SC complexity. This interaction (Figure 4, right) shows a peculiar behavior. Increasing the time between corrections from 1 to 4 periods has a similar impact on the dynamic performance of the three SCs under analysis (the $T B C-B w S l$ curves are characterized by identical slopes). In addition, this impact is very low (i.e., average increase of $4.6 \%$ on $B w S l)$. However, by increasing the time between corrections from 4 to 7 periods, we note very different behaviors between the analyzed SCs (a $B w S l$ increase of $16.78 \%$ for the LCSC and a $B w S l$ increase of $79.69 \%$ for the HCSC). This interaction is summarized in the next finding:

- The impact of the time between corrections on performance significantly depends on the structural complexity of the SC. More specifically, as the structural complexity increases, the SC becomes more sensitive to the configuration of the inventory cycle counting policy. 


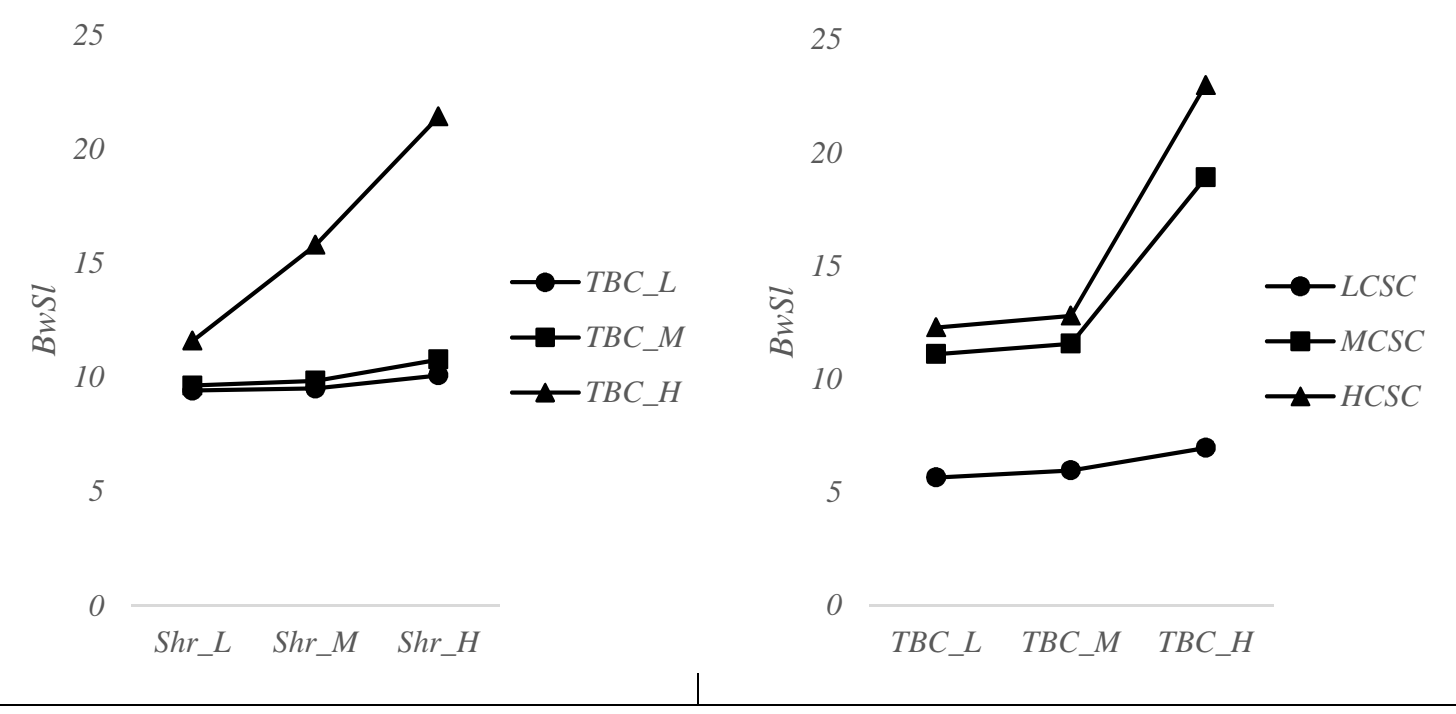

Figure 4. Interaction plots: average $B w S l$ for the different experimental factors.

\subsubsection{Time between corrections and lead time variability}

The interaction between the time between corrections and the lead time variability is shown in Figure 5. We estimate that an increase in the time between corrections from 1 to 4 periods results in an average $B w S l$ increase of $\sim 5 \%$, regardless of the lead time variability. However, an increase in the time between corrections from 4 to 7 periods has a significantly higher impact on $\mathrm{BwSl}$, and in this case, the interaction between the two factors is more relevant. We estimate a $B w S l$ increase of $68.4 \%$ in SCs with high lead time variability and a $\mathrm{BwSl}$ increase of $53.38 \%$ in SCs with low lead time variability. The next finding summarizes this interaction:

- The impact of the time between corrections on performance depends on the lead time variability. More specifically, as the lead time variability increases, the SC becomes more sensitive to the configuration of the inventory cycle counting policy. 


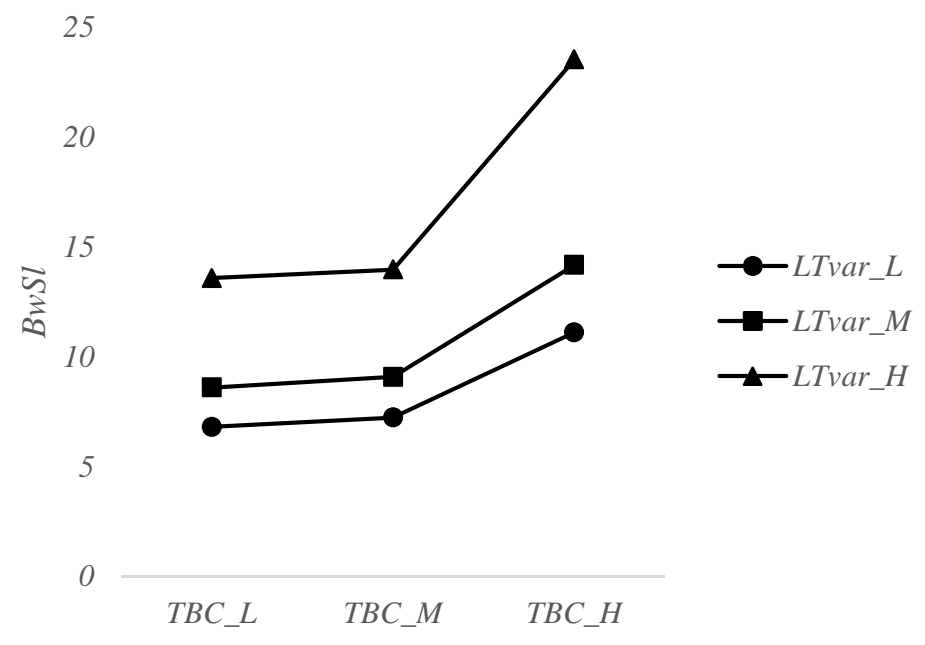

Figure 5. Interaction plot: average $B w S l$ for the different experimental factors.

We present a summary of findings in Table 4. A scale of greys has been used to identify the intensity of the interactions. Light greys represent lower-intensity interactions, while dark greys represent higher-intensity interactions.

\begin{tabular}{|c|c|c|c|}
\hline & SC complexity & $\begin{array}{l}\text { Lead time } \\
\text { variability }\end{array}$ & $\begin{array}{c}\text { Time between } \\
\text { corrections }\end{array}$ \\
\hline $\begin{array}{l}\text { Inventory } \\
\text { Shrinkage }\end{array}$ & $\begin{array}{l}\text { The detrimental effects of } \\
\text { inventory error on the } \\
\text { bullwhip effect are } \\
\text { exacerbated by the } \\
\text { structural complexity of } \\
\text { the SC. }\end{array}$ & $\begin{array}{l}\text { The detrimental effects of } \\
\text { inventory error on the } \\
\text { bullwhip effect are } \\
\text { exacerbated by the lead } \\
\text { time variability. }\end{array}$ & \multirow{2}{*}{$\begin{array}{l}\text { The impact of the inventory error on } \\
\text { the bullwhip effect strongly depends } \\
\text { on the time between corrections. } \\
\text { More specifically, long periods } \\
\text { between inventory corrections } \\
\text { increase the vulnerability of the SC } \\
\text { to the IRI caused by inventory } \\
\text { shrinkage. }\end{array}$} \\
\hline $\begin{array}{l}\text { Time } \\
\text { between } \\
\text { corrections }\end{array}$ & $\begin{array}{l}\text { The benefits that result } \\
\text { from the adoption of an } \\
\text { inventory cycle counting } \\
\text { policy can be diminished } \\
\text { by the SC complexity. }\end{array}$ & $\begin{array}{l}\text { The benefits that result } \\
\text { from the adoption of an } \\
\text { inventory cycle counting } \\
\text { policy can be diminished } \\
\text { by the lead time variability. }\end{array}$ & \\
\hline
\end{tabular}

Table 4. Summary of findings.

\subsection{Sensitivity analysis on structural complexity}

In order to enhance the analyzed simulation models and extend the applicability of the results obtained, we perform a sensitivity analysis (see, e.g., Kleijnen 2005) on the previously analyzed divergent SCs. More specifically, we adopt a scenario decomposition method (Borgonovo and Plischke 2016). According to Tietje (2005), the 
scenarios must be consistent, diverse and small in number. Hence, we use a constant number of Retailers and modify the distribution of nodes in the intermediate echelons, thus generating three new divergent SC configurations characterized by different (exemplary) distribution topologies.

1) Early arborescently (EA) SC: The divergence occurs at the upstream level, i.e., Factory provides to a number of Distributors that is equal to the number of Wholesalers and Retailers of the SC.

2) Late arborescently (LA) SC: The divergence occurs at the downstream level, i.e., a serial structure from Factory to Wholesaler and divergence at the Retailer stage.

3) Intermediate arborescently (IA) SC: The divergence is spread from the upstream to the downstream levels in the SC.

First, we focus on the MCSC scenario (see Figure 6) and study the interactions between the IRI factors (i.e., Shr and $T B C$ ) and the different SC variations, i.e., MCSC-EA, MCSC-IA, MCSC-LA, and the original SC analyzed in the previous section, i.e., the MCSC. To do so, we run a new set of experiments and perform an ANOVA.

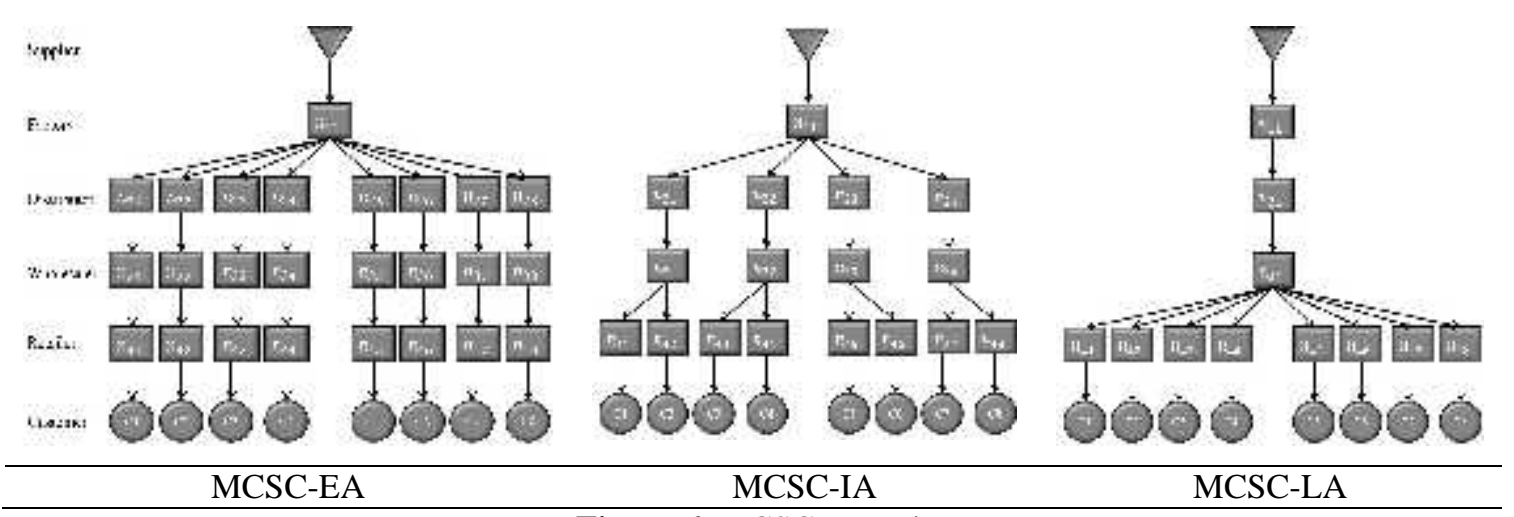

Figure 6. MCSC scenario.

We select the identical three levels for $S h r$ and $T B C$ adopted in the previous design of the experiment (see Table 2). Moreover, to simplify the analysis, we exclude the lead time variance as a factor, and we fix this value to LTvar $=4$ periods $^{2}$. Therefore, we have a total of $3(S h r) \times 3(T B C) \times 4(S C)=36$ experiments. As in the previous analysis, we run 30 replications of each experiment. 
Table 5 shows the results of the ANOVA. The SC configuration has a significant impact on the bullwhip effect ( $F$-ratio 6,716.527), with the MCSC-EA variant showing the lowest bullwhip effect and the MCSC-LA showing the highest bullwhip effect. The effect of $S h r$ and $T B C$ on the bullwhip effect in the SCs under analysis is very similar: even though the interactions $S h r^{*} S C$ and $T B C * S C$ are significant $(p<0.001)$, they have a very low impact on the bullwhip effect (see $F$-ratio in Table 5). This phenomenon can be seen in the interaction plots (Figure 7). It can be observed that the $S h r$ and $T B C$ curves are almost parallel for all SC distributions (i.e., a given increase/decrease of Shr or $T B C$ will result in a similar increase/decrease of the $B w S l)$, thus confirming the low impact of the interactions. In summary, the MCSC structures show similar bullwhip trends under IRI.

\begin{tabular}{llllll}
\hline Source & $D F$ & $S S$ & $M S$ & $F$ & $p$ \\
\hline Adjusted Model & 35 & $36,227.399$ & $1,035.069$ & $3,212.244$ & $<0.001$ \\
Shr & 2 & $5,579.377$ & $2,789.689$ & $8,657.552$ & $<0.001$ \\
$S C$ & 3 & $6,492.719$ & $2,164.240$ & $6,716.527$ & $<0.001$ \\
$T B C$ & 2 & $18,534.260$ & $9,267.130$ & $28,759.721$ & $<0.001$ \\
$S h r * S C$ & 6 & 45.034 & 7.506 & 23.293 & $<0.001$ \\
$S h r * T B C$ & 4 & $5,466.311$ & $1,366.578$ & $4,241.054$ & $<0.001$ \\
$T B C * S C$ & 6 & 102.341 & 17.057 & 52.934 & $<0.001$ \\
Error & 684 & 220.403 & 0.322 & & \\
Total & 720 & $284,311.879$ & & & \\
\hline \multicolumn{7}{c}{ Tabl 5. ANOVA } & & & \\
\hline
\end{tabular}

Table 5. ANOVA - MCSC scenario.

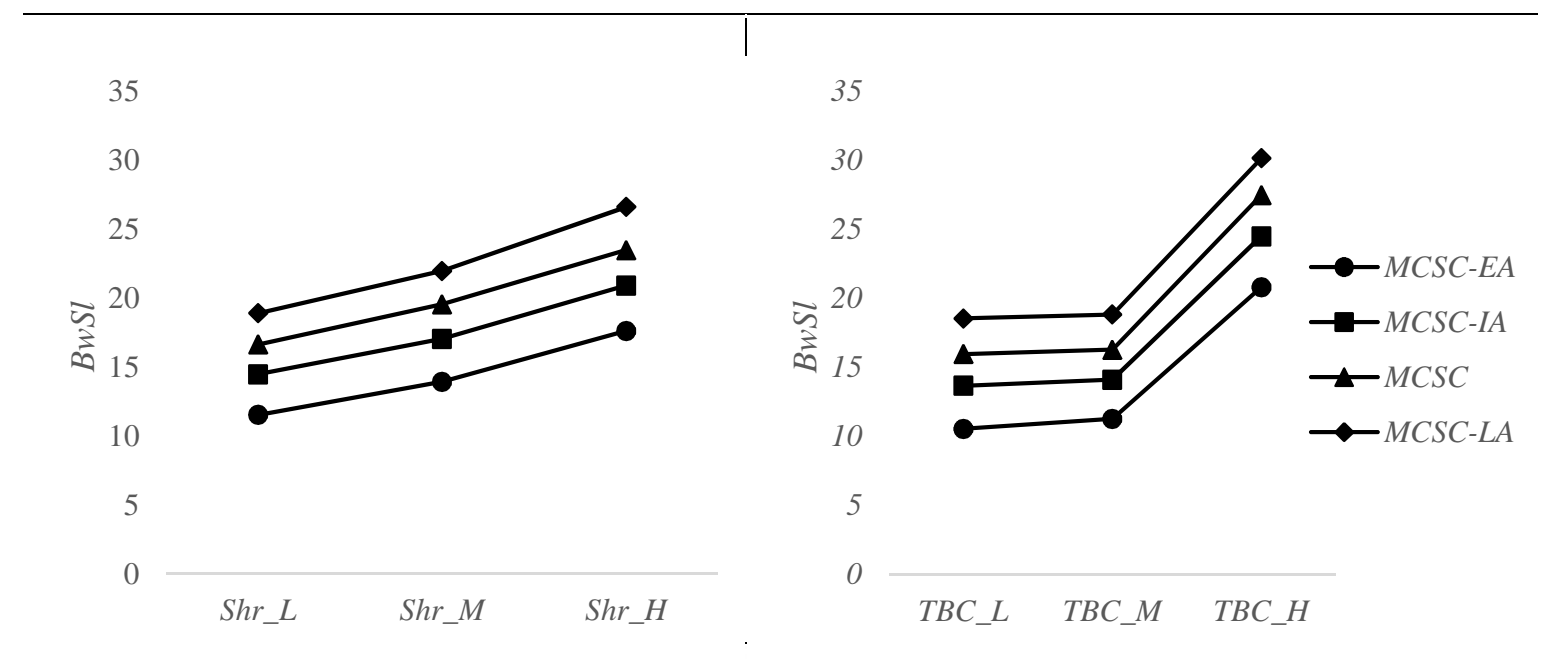

Figure 7. Interaction plots - MCSC scenario. 
Additionally, we perform a similar analysis for the HCSC scenario. Following a similar procedure to that for the MCSC scenario, we generate three new SC distributions, which are shown in Figure 8.

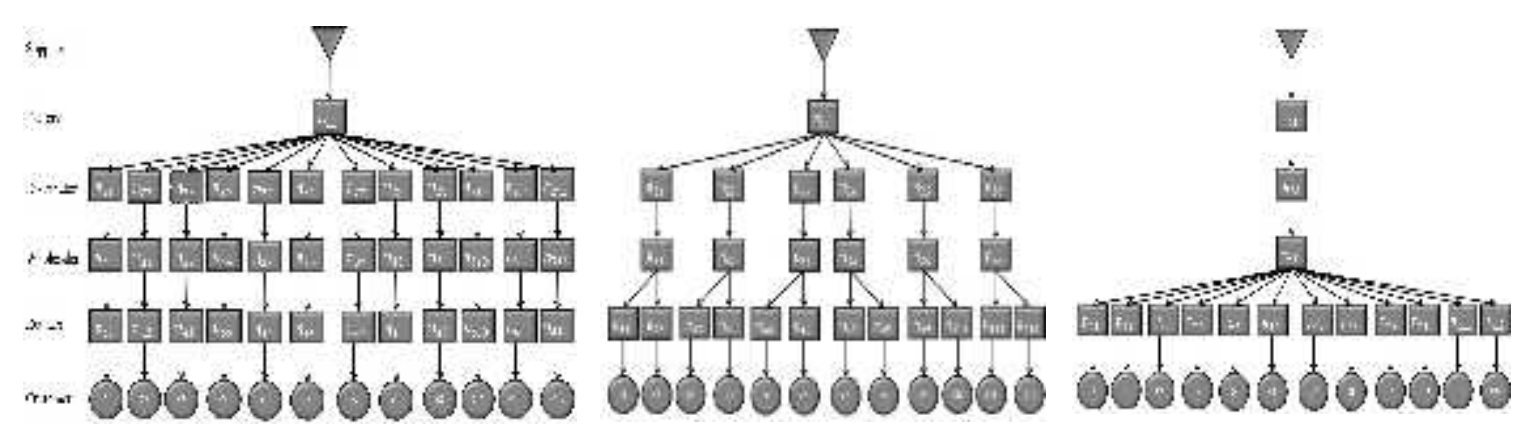

HCSC-EA

HCSC-IA

HCSC-LA

Figure 8. HCSC scenario.

Adopting the analogous DOE of the MCSC scenario, we perform an ANOVA on the new set of 36 experiments and present the results obtained in Table 6. As in the MCSC analysis, we report very low $F$-ratios for the interactions between the pair [Shr,TBC] and the different SC variations. Moreover, the interaction plots in Figure 9 show very similar Shr and TBC curves for the different SCs. Consequently, we conclude with the following finding:

- The impact of shrinkage and time between corrections on the bullwhip effect for different variations of a divergent SC with a given number of echelons and retailers is almost identical.

\begin{tabular}{llllll}
\hline Source & $D F$ & $S S$ & $M S$ & $F$ & $p$ \\
\hline Adjusted Model & 35 & $77,254.012$ & $2,207.257$ & $4,144.017$ & $<0.001$ \\
Shr & 2 & $14,181.347$ & $7,090.674$ & $13,312.389$ & $<0.001$ \\
$S C$ & 3 & $12,948.657$ & $4,316.219$ & $8,103.488$ & $<0.001$ \\
$T B C$ & 2 & $37,467.888$ & $18,733.944$ & $35,172.055$ & $<0.001$ \\
$S h r * S C$ & 6 & 103.578 & 17.263 & 32.411 & $<0.001$ \\
$S h r * T B C$ & 4 & $12,314.423$ & $3,078.606$ & $5,779.930$ & $<0.001$ \\
$T B C * S C$ & 6 & 217.605 & 36.267 & 68.090 & $<0.001$ \\
Error & 684 & 364.324 & .533 & & \\
Total & 720 & $428,795.805$ & & & \\
\hline
\end{tabular}

Table 6. ANOVA - HCSC scenario. 

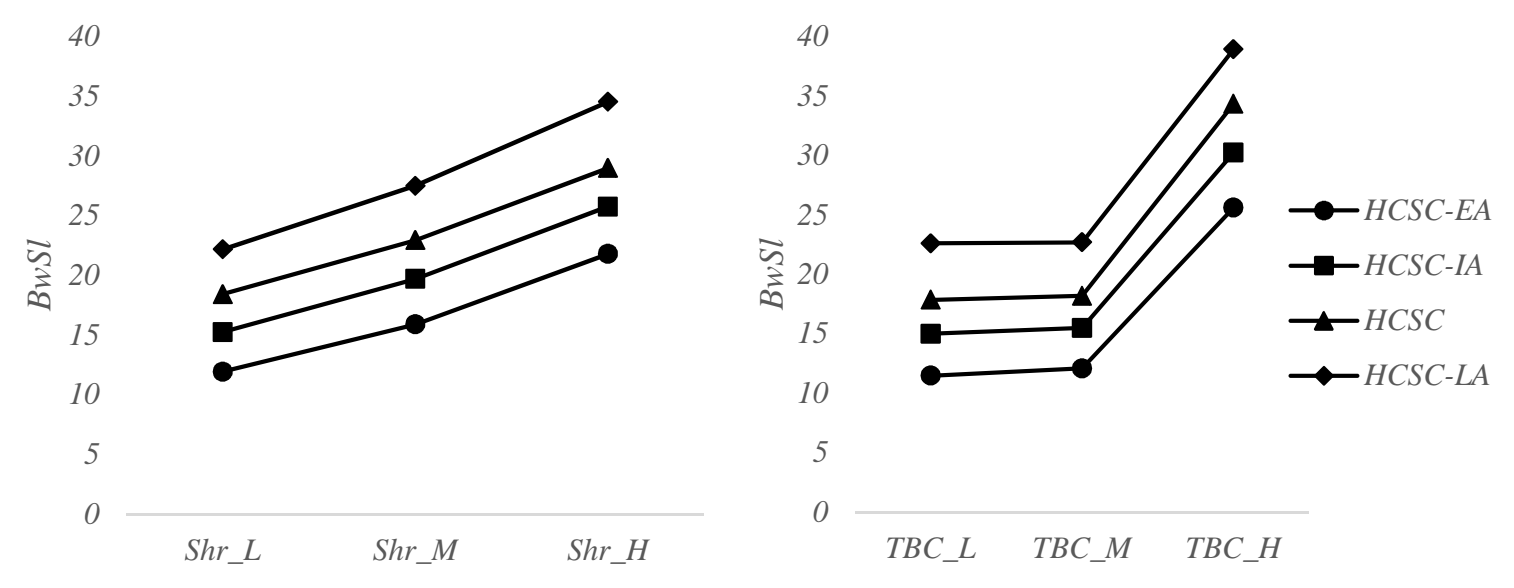

Figure 9. Interaction plots - HCSC scenario.

Finally, in Figure 10, we present a comparison between the pairs [Medium Complexity, High Complexity] of the Shr curves for each of the SC distributions under analysis. It should be noted that, for any given SC distribution, the Shr curves show higher slopes for the SC with High Complexity than for the SC with Medium Complexity. The same occurs when we look at the $T B C$ curves. Thus, the sensitivity analysis confirms the findings obtained in the previous section regarding the interaction between the complexity of the SC and IRI factors.

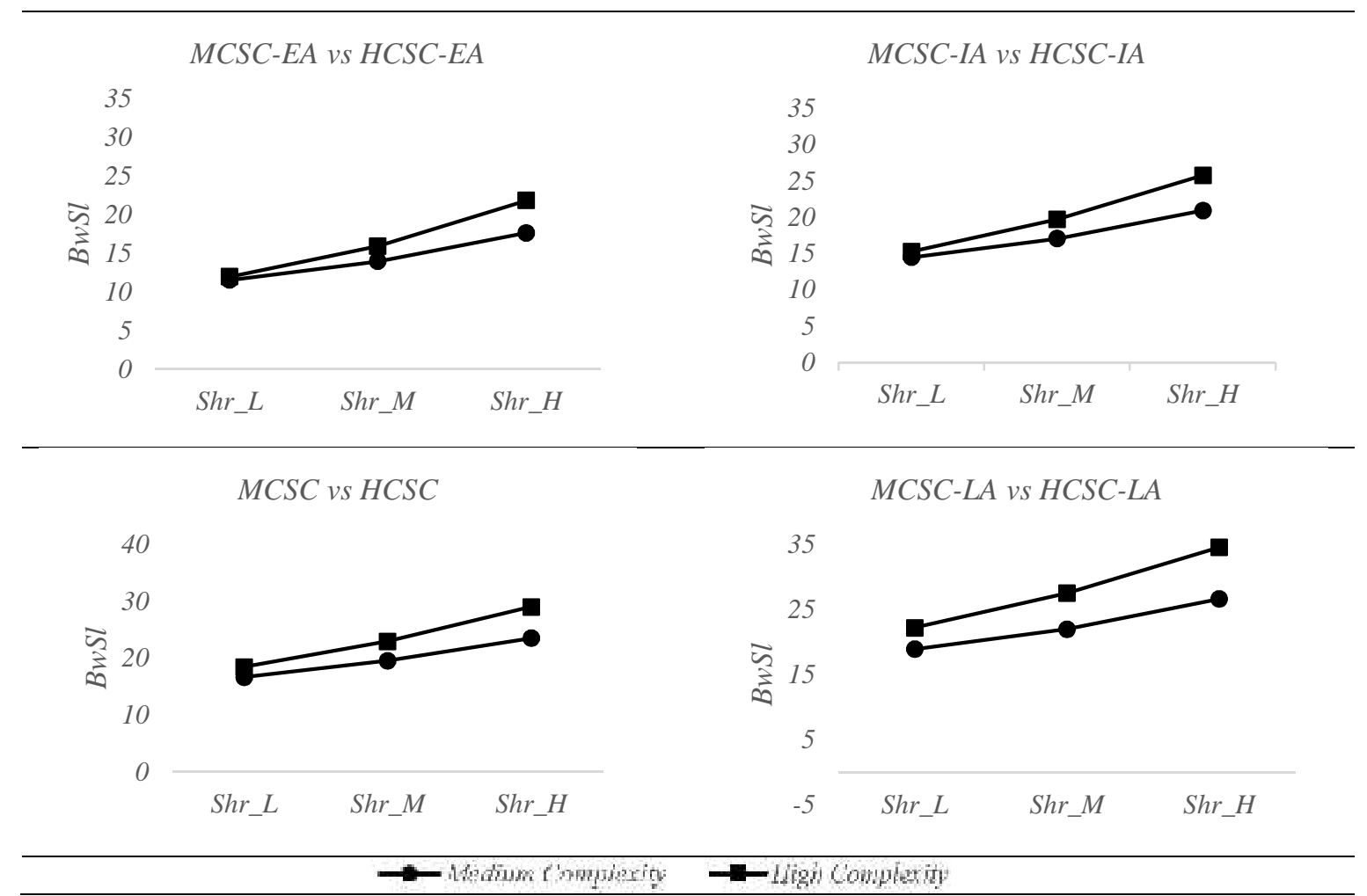

Figure 10. MCSC vs HCSC. 


\section{MANAGERIAL IMPLICATIONS}

In this section, we discuss managerial implications obtained from our work. More specifically, we provide recommendations that may help managers to better understand IRI and to limit its consequences. In particular, we focus on how IRI-corrective and preventive strategies can be improved in light of our results.

\subsection{Corrective strategies for IRI}

Standard corrective techniques for IRI include inventory audits, such as cycle counting (de Kok et al. 2008, Kok and Shang 2014). Adopting a low frequency of inventory audits (i.e., long time between corrections) cannot effectively reduce the detrimental effects of IRI, particularly if the SC experiences a considerable magnitude of inventory errors. However, adopting a high frequency improves the effectiveness of this corrective strategy and may drastically reduce the sensitivity of the SC to IRI. Nevertheless, inventory audits are expensive and disruptive (Get al., 2010) and may involve important costs for each company.

Consequently, this frequency has to be based on a context-related trade-off analysis between the costs derived from IRI occurrence and the costs of inventory audits. Usually, in the IRI literature, this trade-off is based on a cost function that calculates the frequency of counts in order to minimize the total cost and ensure an acceptable stockout level or a sufficient buffer stock at a single or two-echelon system (see, e.g., Rossetti et al. 2010, Agrawal and Sharda 2012, Kok and Shang 2007, 2014, Fan et al. 2014). However, this work suggests that the effectiveness of inventory audits should also consider other relevant factors, i.e., lead time variability and SC structure. Both factors seem to amplify the detrimental consequences of IRI. Under an identical magnitude of inventory error, a complex SC with higher lead time variability is more sensitive to the frequency of inventory audits than a simpler SC with low lead time variability. Thus, the risk related to decision making on the frequency of a cycle counting is more relevant in the former scenario. This implies that SCs characterized by noteworthy geographical dispersion (i.e., high lead time variability) and by multiple retailers and distribution centers (i.e., high SC structural complexity) have to pay particular attention to potential costs generated by the time-varying phenomenon when adopting and devising inventory audit policies. In such cases, they may increase the 
frequency of inventory audits, even in spite of a superior investment in IRI-corrective strategies in order to further prevent unnecessary costs.

\subsection{Preventive strategies for IRI}

Corrective strategies only offer a costly ex post remedy to the inventory error occurrences when IRI is already present among SC members, and the potential detrimental consequences spread along the chain. As a palliative measure, it may reduce the effect of inventory errors, but by nature, it is not designed to eradicate the IRI problem. Preferably, investments may be focused on preventive strategies in order to reduce the root causes of errors. Improvements in workforce education, product and shipment labeling, the shelving and storage of items, the foolproofing of procedures, information technology, and product tagging, among others (see, e.g., DeHoratious and Raman 2008, Gel et al. 2010,, Zhu et al., 2011, Kull et al., 2011; Shin and Eksioglu, 2015), represent useful techniques to prevent IRI occurrences. However, it has been shown that inventory errors cannot be completely eliminated, even by adopting innovative prevention strategies, such as RFID technology (Zhou 2009).

This work indicates that in simple SCs with low lead time variability, after the implementation of a preventive technique, the impact of any potential residual errors may be insignificant. However, in complex SCs with high lead time variability, identical residual errors can undermine the investment in IRI-preventive strategies. Therefore, to guarantee investment in such prevention strategies, managers may consider developing complementary preventative strategies.

First, they may focus on production and operations approaches devoted to smoothing lead time variability. Among these, process simplification using lean and six sigma methodologies and contracting superior transportation outsourcing services can be considered. Another possible solution is the introduction of new warehouses or distribution centers to reduce distances and lead time variability among SC partners. However, this approach can generate an unwanted effect because the inclusion of such centers may increase the SC complexity and exacerbate the impact of inventory errors as well.

Moreover, as self-interested parties and the lack of coordination among SC members can exacerbate lead time variability, the adoption of consolidated best practices, such as collaboration, integration and the sharing of benefits among partners, would yield 
noticeable benefits (Fawcett et al. 2011, Audy et al. 2012, Chan et al. 2012, Fan et al. $2014,2015)$. Such approaches would not only facilitate the implementation of specific preventive strategies, such as product tracking, and stimulate members to correctly implement such strategies, but also improve their efficacy by reducing lead time variability.

Finally, to limit the amplification effect of SC complexity on IRI consequences, managers may implement SC design and reengineering approaches devoted to the simplification of the SC structure, such as a structural lean strategy (Holweg and Christopher 2011, Cannella et al. 2015) (i.e., reducing the number of retailers or wholesalers). However, this approach is not always implementable due to the technological and operational limitations of many SC processes.

\section{CONCLUSIONS AND LIMITATIONS}

This paper analyses the impact of IRI on the dynamic performance of both simple and complex SC structures under a variety of operational parameter conditions. We model and simulate three different SCs characterized by an increasing level of structural complexity under different IRI scenarios. To emulate these scenarios, we model a gap between the physical inventory and the inventory record and introduce a shrinkage error into the inventory system of each SC member. We adopted a full-factorial experimental design and studied the following four factors: (1) the level of inventory errors, (2) the structural complexity of the SC, (3) the lead time variance and (4) the time between inventory corrections. In order to perform the analysis, we employed a MAS modeling approach. Assumptions and data input for the simulation were obtained from different cases to emulate real-world logistic systems. Dynamic performance was measured through the order rate variance ratio, also known as the bullwhip ratio (Chen et al. 2000), a common metric adopted for the evaluation of SC dynamics.

As a result, we have generated new knowledge regarding the IRI problem in SCs. Among these novel insights is that the detrimental effects of IRI on SC performance are exacerbated by the structural complexity of the SC and lead time variability. Furthermore, we have shed light on the efficacy of IRI-limiting strategies by showing how the benefit provided by these costly policies may be diminished by SC design and operational factors. 
Moreover, we have provided insightful directions to properly apply IRI-corrective strategies. Particularly, we have suggested specific actions to be considered under different SC designs and operational scenarios in order to avoid the risk of annihilating investments in such IRI-avoiding approaches. More specifically, this work has underlined how the risk related to decisions on IRI-limiting strategies may be more relevant for complex SCs with high lead time uncertainty. Thus, we have suggested the adoption of complementary approaches in order to improve the efficacy of such strategies by limiting lead time variability (i.e., process simplification using lean and six sigma methodologies, improving transportation outsourcing services, collaboration, integration and the sharing of benefits among partners) and by reducing SC complexity (i.e., lean structure design and reengineering $\mathrm{SC}$ ).

Finally, this study also presents important novel implications regarding the impact that two common modeling assumptions have on the accuracy of what-if analysis. In reality, assuming a serial or mono-echelon SC and/or deterministic lead times while providing important benefits in terms of modeling simplification may produce important differences for the estimation of the impact of IRI. Indeed, our study shows relevant interactions between the mentioned assumptions, the impact of IRI and the frequency of inventory audits on SC performance. Therefore, it is beneficial to adopt more realistic SC models in order to increase the consistency of simulation outputs and to gain more precise and novel insights on the impact of IRI in SCs.

This analysis presents some limitations that may create room for improvement and further research. The first limitation is related to the SCs under analysis. In order to establish the relationship between the structural complexity of SCs and inventory errors, we focused on one SC topology (distribution SCs), as in Dominguez et al. (2015a). Therefore, the present study can be extended by analyzing IRI in different SC configurations, such as convergent (assembly type) or conjoined (mixed convergentdivergent) SCs. Convergent SCs may show different results from those observed for divergent SCs because there are fewer companies at lower echelons of the chain that create the instabilities caused by inventory errors. Additionally, these instabilities are shared among many members in the upper echelons. For similar reasons, the analysis of inventory errors in conjoined SCs should be addressed.

Analogously, we analyzed a specific inventory error, i.e., shrinkage. However, other types of both transaction-independent and -dependent errors (e.g., unreliable suppliers, 
internal movement of materials, incorrect deliveries and picking misplaced items) were not considered in our analysis. Further works may extend our analysis to study the impact of such errors.

Another important limitation refers to the assumption that all companies present the same level of inventory error. We have made this simplification in order to clarify the different aspects of the relationship between the inventory error problem, the configuration of the SC and lead time variability. However, it is reasonable to consider that retailers face a different level of inventory than do their upstream partners. In future works, it would be of interest to analyze the effect of distributed inventory errors.

A very interesting phenomenon is the significant jump in the bullwhip effect reported for high values of the time between corrections. As the time between corrections increases, the discrepancy between physical inventory and recorded inventory at the moment of inventory alignment increases as well. Thus, when a generic member aligns his inventory, he generates a "pulse" in the order pattern. This pulse exponentially amplifies the order variability upstream in the SC. This phenomenon could be the reason for the strong non-linearity observed for the bullwhip slope as the time between corrections increases. The determination of a possible threshold value for the time between corrections and how operational and structural characteristics of the SC may impact this value would be valuable for SC managers and requires additional research.

Moreover, in this work, we have adopted well-established modeling assumptions and obtained theoretical distributions for data input (Evers and Wan 2012) to emulate the probabilistic nature of several SC factors on the basis of other relevant empirical, analytical, and what-if analyses. However, this work lacks a grounded empirical method. Further work may employ a multi-method approach (Kull et al. 2013) using both empirical and simulated IRI data to validate and deepen our contribution.

Finally, in the previous section, we suggested that managers of upstream echelons of the SC may incentivize companies at lower echelons (retailers) to introduce and properly apply preventive/corrective strategies. However, as the number of retailers increase, investments in such strategies may increase as well. Hence, it would be interesting to address how the benefits under different scenarios of SC configurations may be altered in order to design proper incentives. 


\section{ACKNOWLEDGEMENTS}

We are grateful to the anonymous referees and the editorial team as their suggestions allowed us to improve earlier versions of this manuscript. This research was supported by the European Commission/Andalusian Agency of Knowledge (Talentia Marie Skłodowska-Curie Cofund Fellow), by the Portuguese Foundation for Science and Technology [grant SFRH/BPD/108491/2015] and by the Spanish Ministry of Science and Innovation, under project ADDRESS with reference DPI2013-44461-P/DPI and fellowship Juan de la Cierva Incorporación.

\section{REFERENCES}

Agrawal, P.M., Sharda, R. 2012. Impact of frequency of alignment of physical and information system inventories on out of stocks: A simulation study. International Journal of Production Economics, 136 (1), 45-55.

Annarelli, A., \& Nonino, F. (2016). Strategic and operational management of organizational resilience: Current state of research and future directions. Omega 62, 1-18

Audy, J.-F., Lehoux, N., D'Amours, S., Rönnqvist, M. 2012. A framework for an efficient implementation of logistics collaborations. International Transactions in Operational Research, 19 (5), 633-657.

Bhattacharya, R., Bandyopadhyay, S. 2011. A review of the causes of bullwhip effect in a supply chain. The International Journal of Advanced Manufacturing Technology, 54(9-12), 1245-1261.

Borgonovo, E., Plischke, E. 2016. Sensitivity analysis: a review of recent advances. European Journal of Operational Research, 248(3), 869-887.

Bruccoleri, M., Cannella, S., La Porta, G. 2014. Inventory record inaccuracy in supply chains: the role of workers' behavior. International Journal of Physical Distribution \& Logistics Management, 44 (10), 796- 819

Cannella S., Framinan, J.M., Bruccoleri, M., Barbosa-Póvoa, A.P., Relvas, S. 2015. The effect of Inventory Record Inaccuracy in Information Exchange Supply Chains. European Journal of Operational Research, 243 (1), 120-129.

Cannella, S. 2014. Order-Up-To policies in Information Exchange supply chains. Applied Mathematical Modelling, 38 (23), 5553-5561.

Cannella, S., Barbosa-Povoa, A.P., Framinan J.M., Relvas S. 2013. Metrics for bullwhip effect analysis. Journal of the Operational Research Society, 64, 1-16.

Cardoso, S. R., Barbosa-Póvoa, A. P., Relvas, S., \& Novais, A. Q. 2015. Resilience metrics in the assessment of complex supply-chains performance operating under demand uncertainty. Omega, 56, 5373.

Chan, F.T.S, Prakash, A. 2012. Inventory management in a lateral collaborative manufacturing supply chain: a simulation study. International Journal of Production Research, 50 (16), 4670-4685.

Chan, H.-L., Choi, T.-M., Hui, C.-L. 2012. RFID versus bar-coding systems: Transactions errors in health care apparel inventory control. Decision Support Systems, 54 (1), 803-811.

Chen, S., Wang, H., Xie, Y., \& Qi, C. 2014. Mean-risk analysis of radio frequency identification technology in supply chain with inventory misplacement: Risk-sharing and coordination. Omega, 46, 86-103. 
Chatfield D.C., Pritchard A.M. 2013. Returns and the bullwhip effect. Transportation Research Part E: Logistics and Transportation Review, 49 (1), 159-175.

Chatfield, D., Harrison, T., Hayya, J. 2001. SISCO: A supply chain simulation tool utilizing Silk ${ }^{\mathrm{TM}}$ and XML. Winter Simulation Conference Proceedings, 1, 614-622.

Chatfield, D.C. 2013. Underestimating the bullwhip effect: a simulation study of the decomposability assumption. International Journal of Production Research, 51 (1), 230-244.

Chatfield, D.C., Hayya, J.C., Cook, D.P. 2012. Stockout propagation and amplification in supply chain inventory systems. International Journal of Production Research, 51 (5), 1491-1507.

Chatfield, D.C., Kim, J.G., Harrison, T.P., Hayya, J.C. 2004. The bullwhip effect - Impact of stochastic lead time, information quality, and information sharing: A simulation study. Production and Operations Management, 13 (4), 340-353.

Chen, F., Drezner, Z., Ryan, J.K., Simchi-Levi, D. 2000. Quantifying the bullwhip effect in a simple supply chain: The impact of forecasting, lead times, and information. Management Science, 46 (3), 436-443.

Chen, H.-H. 2012. Simulating analysis of complex supply chain networks invulnerability. Lecture Notes in Electrical Engineering, 154, 1229-1235.

Christopher, M., Holweg, M. 2011. "Supply Chain 2.0": Managing supply chains in the era of turbulence. International Journal of Physical Distribution and Logistics Management, 41 (1), 63-82.

Christopher, M., Ryals, L.J. 2014. The supply chain becomes the demand chain. Journal of Business Logistics, 35 (1), 29-35.

Ciancimino E., Cannella S., Bruccoleri M., Framinan, J.M. 2012. On the bullwhip avoidance phase: the Synchronised Supply. European Journal of Operational Research, 221 (1), 49-63

Croson, R., Donohue, K. 2006. Behavioral causes of the bullwhip effect and the observed value of inventory information. Management Science, 52 (3), 323-336

Croson, R., Donohue, K., Katok, E., Sterman, J. 2014. Order stability in supply chains: coordination risk and the role of coordination stock. Production and Operations Management, 23 (2), 176-196.

Dai, H., Tseng, M.M. 2012. The impacts of RFID implementation on reducing inventory inaccuracy in a multi-stage supply chain. International Journal of Production Economics, 139 (2), 634-641.

de Kok, A.G., van Donselaar, K.H., van Woensel, T. 2008. A break-even analysis of RFID technology for inventory sensitive to shrinkage. International Journal of Production Economics, 112 (2), 521-531.

Dehoratius, N., Mersereau, A.J., Schrage, L. 2008. Retail Inventory Management When Records Are Inaccurate. Manufacturing and Service Operations Management, 10 (2), 257-277.

DeHoratius, N., Raman, A. 2008. Inventory record inaccuracy: An empirical analysis. Management Science, 54 (4), 627-641.

Dejonckheere, J., Disney, S. M., Lambrecht, M. R., Towill, D. R. 2004. The impact of information enrichment on the Bullwhip effect in supply chains: A control engineering perspective. European Journal of Operational Research, 153 (3), 727-750.

Disney, S.M., Lambrecht, M. 2008. On Replenishment Rules, Forecasting, and the Bullwhip Effect in Supply Chains. Foundations and Trends in Technology, Information and Operations Management, 2 (1), 1-80.

Disney, S.M., Naim, M.M., Potter, A. 2004. Assessing the impact of e-business on supply chain dynamics. International Journal of Production Economics, 89 (2), 109-118.

Dominguez, R., Framinan, J.M. 2013. A decisión management tool: modelling the order fulfilment process by multi-agent systems. International Journal of Management and Decision Making, 12 (3), $240-258$.

Dominguez, R., Framinan, J.M., Cannella, S. 2014a. Serial vs. divergent supply chain networks: a comparative analysis of the bullwhip effect. International Journal of Production Research, 52 (7), 2194-2210.

Dominguez, R., Cannella, S., Framinan, J.M. 2014b. On bullwhip-limiting strategies in divergent supply chain networks. Computers and Industrial Engineering, 73 (1), 85-95. 
Dominguez, R., Framinan, J.M., Cannella, S. 2015a. The impact of the supply chain structure on bullwhip effect. Applied Mathematical Modelling, 39, 7309-7325.

Dominguez, R., Cannella, S., Framinan, J.M. 2015b. On returns and network configuration in supply chain dynamics. Transportation Research Part E: Logistics and Transportation Review, 73, 152-167.

Evers, P. T. 1999. Filling customer orders from multiple locations: a comparison of pooling methods. Journal of Business Logistics, 20, 121-140.

Evers, P.T., Wan, X. 2012. Systems analysis using simulation. Journal of Business Logistics, 33 (2), 8089.

Fan, T. J., Chang, X. Y., Gu, C. H., Yi, J. J., Deng, S. 2014. Benefits of RFID technology for reducing inventory shrinkage. International Journal of Production Economics, 147 (part C), 659-665.

Fan, T., Tao, F., Deng, S., \& Li, S. 2015. Impact of RFID technology on supply chain decisions with inventory inaccuracies. International Journal of Production Economics, 159, 117-125.

Fawcett, S.E., Osterhaus, P., Magnan, G.M., Brau, J.C., McCarter, M.W. 2007. Information sharing and supply chain performance: The role of connectivity and willingness. Supply Chain Management, 12 (5), 358-368.

Fawcett, S.E., Wallin, C., Allred, C., Fawcett, A.M., Magnan, G.M. 2011. Information technology as an enabler of supply chain collaboration: A dynamic-capabilities perspective. Journal of Supply Chain Management, 47 (1) , 38-59.

Fleisch, E., Tellkamp, C. 2005. Inventory inaccuracy and supply chain performance: A simulation study of a retail supply chain. International Journal of Production Economics, 95 (3), 373-385.

Forrester, J. 1961. Industrial Dynamics. MIT Press, Cambridge.

Garcia, C. A., Ibeas, A., Herrera, J., Vilanova, R. 2012. Inventory control for the supply chain: an adaptive control approach based on the identification of the lead-time. Omega, 40 (3), 314-327.

Gel, E.S., Erkip, N., Thulaseedas, A. 2010. Analysis of simple inventory control systems with execution errors: Economic impact under correction opportunities. International Journal of Production Economics, 125 (1), 153-166.

Giard, V., Sali, M. 2013. The bullwhip effect in supply chains: a study of contingent and incomplete literature. International Journal of Production Research, 51(13), 3880-3893.

Govindu, R., Chinnam, R.B. 2010. A software agent-component based framework for multi-agent supply chain modeling and simulation. International Journal of Modeling and Simulation, 30 (2), 155-171.

Guertler, B., Spinler, S. 2015. When does operational risk cause supply chain enterprises to tip? A simulation of intra-organizational dynamics. Omega, 57, 54-69.

Gumrukcu, S., Rossetti, M.D., Buyurgan, N. 2008. Quantifying the costs of cycle counting in a twoechelon supply chain with multiple items. International Journal of Production Economics, 116 (2), 263274.

Hardgrave, B.C., Aloysius, J.A., Goyal, S. 2013. RFID-Enabled Visibility and Retail Inventory Record Inaccuracy: Experiments in the Field. Production and Operations Management. 22 (4), 843-856.

Hardgrave, B.C., Aloysius, J.A., Goyal, S. 2013. RFID-Enabled Visibility and Retail Inventory Record Inaccuracy: Experiments in the Field. Production and Operations Management, 22 (4), 843-856.

Hax, A.C., and Candea, D., 1984. Production and Inventory Management. Englewood Cliffs: PrenticeHall.

Heckmann, I., Comes, T., \& Nickel, S. 2015. A critical review on supply chain risk-Definition, measure and modeling. Omega, 52, 119-132.

Heese, H.S. 2007. Inventory record inaccuracy, double marginalization, and RFID adoption. Production and Operations Management, 16 (5), 542-553.

Hinkelmann, K., Kempthorne, O. 1994. Design and Analysis of Experiments: Volume I - Introduction to Experimental Design, Wiley, New York, NY.

Hollinger, R.C., Adams, A. 2010. 2009 National Retail Security Survey Final Report. Report, University of Florida, USA. 
Hussain, M., Drake, P.R., Lee, D.M. 2012. Quantifying the impact of a supply chain's design parameters on the bullwhip effect using simulation and Taguchi design of experiments. International Journal of Physical Distribution and Logistics Management, 42 (10), 947-968.

Hwarng, H.B., Chong, C.S.P., Xie, N., Burgess, T.F. 2005. Modelling a complex supply chain: Understanding the effect of simplified assumptions. International Journal of Production Research, 43 (13), 2829-2872.

Kang, Y., Gershwin, S.B. 2005. Information inaccuracy in inventory systems: Stock loss and stockout. IIE Transactions, 37 (9), 843-859.

Ketzenberg, M.E., Geismar, N., Metters, R., and van der Laan, E. 2013. The Value of Information for Managing Retail Inventory Remotely. Production and Operations Management, 22 (4), 811-825.

Khader, S., Rekik, Y., Botta-Genoulaz, V., Campagne, J.-P. 2014. Inventory management subject to multiplicative inaccuracies. International Journal of Production Research, 52 (17), 5055-5069.

Kleijnen, J. P. C. 2008. Simulation experiments in practice: statistical design and regression analysis. Journal of Simulation, 2(1), 19-27.

Kök, A. G., Shang, K. H. 2007. Inspection and replenishment policies for systems with inventory record inaccuracy. Manufacturing \& Service Operations Management,9 (2), 185-205.

Kök, A.G., Shang, K.H. 2014. Evaluation of cycle-count policies for supply chains with inventory inaccuracy and implications on RFID investments. European Journal of Operational Research, 237 (1), 91-105..

Kull, T. J., Barratt, M., Sodero, A. C., Rabinovich, E. 2013. Investigating the Effects of Daily Inventory Record Inaccuracy in Multichannel Retailing. Journal of Business Logistics, 34 (3), 189-208.

Kwak, J.K., Gavirneri S. 2014. Impact of information errors on supply chain performance. Journal of the Operational Research Society, 66 (2), 288-298.

Lee, H. L., Padmanabhan, V., Whang, S., 1997. Information distortion in a Supply Chain: The Bullwhip effect. Management Science, 43 (4), 546-558.

Lee, H., Özer, Ö. 2007. Unlocking the value of RFID. Production and Operations Management, 16 (1), 40-64.

Li, G., Xuan, Q., Song, Z., Jin, X. 2010a. Complex supply networks evolving model: Complex networks perspective. Proceedings of 2010 IEEE International Conference on Intelligent Systems and Knowledge Engineering, ISKE 2010, art. no. 5680780, 511-516.

Li, G., Yang, H., Sun, L., Ji, P., Feng, L. 2010b. The evolutionary complexity of complex adaptive supply networks: A simulation and case study. International Journal of Production Economics, 124 (2), 310330 .

Li, Q., Disney, S. M., Gaalman, G. (2014). Avoiding the bullwhip effect using Damped Trend forecasting and the Order-Up-To replenishment policy. International Journal of Production Economics, 149, 3-16.

Li, T., Zhang, H. 2015. Information sharing in a supply chain with a make-to-stock manufacturer. Omega, 50, 115-125.

Li, Y., Zhen, X., Qi, X., \& Cai, G. G. 2016. Penalty and financial assistance in a supply chain with supply disruption. Omega, 61, 167-181.

Long, Q., Lin, J., Sun, Z., 2011. Modeling and distributed simulation of supply chain with a multi-agent platform. International Journal of Advanced Manufacturing Technology, 55 (9-12), 1241-1252.

Long, Q., Zhang, W. 2014. An integrated framework for agent based inventory-production-transportation modeling and distributed simulation of supply chains. Information Sciences, 277, 567-581.

Mersereau, A.J. 2013. Information-Sensitive Replenishment when Inventory Records Are Inaccurate. Production and Operations Management, (22) 4, 792-810.

Merzifonluoglu, Y. 2015. Risk averse supply portfolio selection with supply, demand and spot market volatility. Omega, 57, 40-53.

Metzger, C., Thiesse, F., Gershwin, S., Fleisch, E. 2013. The impact of false-negative reads on the performance of RFID-based shelf inventory control policies. Computers and Operations Research, 40 (7), 1864-1873. 
Nachtmann, H., Waller, M.A., Rieske, D.W. 2010. The impact of point-of-sale data inaccuracy and inventory record data errors. Journal of Business Logistics, 31 (1), 149-158.

Pathak, S.D., Day, J.M., Nair, A., Sawaya, W.J., Kristal, M.M. 2007. Complexity and adaptivity in supply networks: Building supply network theory using a complex adaptive systems perspective. Decision Sciences, 38 (4), 547-580.

Rabinovich, E., Cheon, S. 2011. Expanding horizons and deepening understanding via the use of secondary data sources. Journal of Business Logistics, 32 (4), 303-316.

Rekik, Y., Sahin, E. 2012. Exploring inventory systems sensitive to shrinkage - Analysis of a periodic review inventory under a service level constraint. International Journal of Production Research, 50 (13), 3529-3546.

Rekik, Y. 2011. Inventory inaccuracies in the wholesale supply chain. International Journal of Production Economics, 133 (1), 172-181

Rekik, Y., Sahin, E., Dallery, Y. 2009. Inventory inaccuracy in retail stores due to theft: An analysis of the benefits of RFID. International Journal of Production Economics, 118 (1), 189-198.

Rekik, Y., Jemai, Z., Sahin, E., Dallery, Y. 2007. Improving the performance of retail stores subject to execution errors: Coordination versus RFID technology. OR Spectrum, 29 (4) , pp. 597-626.

Rossetti, M. D., Buyurgan, N., Bhonsle, A., \& Gumrukcu, S. 2010. An analysis of the effect of inventory record inaccuracy in a two-echelon supply chain. International Journal of Inventory Research, 1(2), 174-208.

Sahin, E., Buzacott, J., Dallery, Y. 2009. Analysis of a newsvendor which has errors in inventory data records. European Journal of Operational Research, 188 (2), 370-389.

Sahin, E., Dallery, Y. 2009. Assessing the impact of inventory inaccuracies within a Newsvendor framework. European Journal of Operational Research, 197 (3), 1108-1118.

Sarac, A., Absi, N., Dauzère-Pérès, S. 2015. Impacts of RFID technologies on supply chains: a simulation study of a three-level supply chain subject to shrinkage and delivery errors. European Journal of Industrial Engineering, 9 (1), 27-52.

Sarac, A., Absi, N., Dauzre-Prs, S. 2010. A literature review on the impact of RFID technologies on supply chain management. International Journal of Production Economics, 128 (1), 77-95.

Sari, K. 2008. Inventory inaccuracy and performance of collaborative supply chain practices. Industrial Management and Data Systems, 108 (4), 495-509.

Sari, K. 2015. Investigating the value of reducing errors in inventory information from a supply chain perspective. Kybernetes, 44 (2), 176 - 185

Shan, J., Yang, S., Yang, S., Zhang, J. 2014. An empirical study of the bullwhip effect in china. Production and Operations Management 23 (4), 537-551

Shin, S., Eksioglu, B. 2015. An empirical study of RFID productivity in the US retail supply chain. International Journal of Production Economics, 163, 89-96.

Stank, T.P., Dittmann, J.P., Autry, C.W. 2011. The new supply chain agenda: A synopsis and directions for future research. International Journal of Physical Distribution and Logistics Management, 41 (10), 940-955.

Sterman, J. 1989. Modeling managerial behavior: misperceptions of feedback in a dynamic decision making experiment. Management Science, 35 (3), 321-339.

Sucky, E. 2009. The Bullwhip Effect in Supply Chains - An Overestimated Problem?. International Journal of Production Economics, 118 (1), 311-322.

Surana, A., Kumara, S., Greaves, M., Raghavan, U.N. 2005. Supply-chain networks: A complex adaptive systems perspective. International Journal of Production Research, 43 (20), 4235-4265.

Swaminathan, J.M., Smith, S.F., Sadeh, N.M. 1998. Modeling supply chain dynamics: a multi-agent approach. Decision Sciences, 29 (3), 607-631.

Talluri, S., Kull, T.J., Yildiz, H., Yoon, J. 2013. Assessing the efficiency of risk mitigation strategies in supply chains. Journal of Business Logistics, 34 (4), 253-269.

Thiel, D., Hovelaque, V., Thi Le Hoa, V. 2010. Impact of inventory inaccuracy on service-level quality in $(\mathrm{Q}, \mathrm{R})$ continuous-review lost-sales inventory models. International Journal of Production Economics, 
123 (2), 301-311.

Tietje, O. 2005. Identification of a small reliable and efficient set of consistent scenarios. European Journal of Operational Research, 162(2), 418-432.

Trapero, J.R., Kourentzes, N., Fildes, R. 2012. Impact of information exchange on supplier forecasting performance. Omega, 40 (6), 738-747.

Turrisi, M., Bruccoleri, M., Cannella, S. 2013. Impact of reverse logistics on supply chain performance. International Journal of Physical Distribution \& Logistics Management 43 (7), 564 - 585.

Uçkun, C., Karaesmen, F., Savas, S. 2008. Investment in improved inventory accuracy in a decentralized supply chain. International Journal of Production Economics, 113 (13), 546-566.

Waller, M.A., Fawcett, S.E. 2014. Click here to print a maker movement supply chain: How invention and entrepreneurship will disrupt supply chain design. Journal of Business Logistics, 35 (2), 99-102

Wan, X., Evers, P.T. 2011. Supply chain networks with multiple retailers: A test of the emerging theory on inventories, stockouts, and bullwhips. Journal of Business Logistics, 32 (1), 27-39.

Wang, X., Disney, S. M. 2015. The bullwhip effect: Progress, trends and directions. European Journal of Operational Research. DOI: 10.1016/j.ejor.2015.07.022.

Xu, J., Jiang, W., Feng, G., Tian, J. 2012. Comparing improvement strategies for inventory inaccuracy in a two-echelon supply chain. European Journal of Operational Research, 221 (1), 213-221.

Zissis, D., Ioannou, G., Burnetas, A. 2015. Supply chain coordination under discrete information asymmetries and quantity discounts. Omega, 53, 21-29.

Zhou, W. 2009. RFID and item-level information visibility. European Journal of Operational Research 198 (1), 252-258.

Zhu, X., Mukhopadhyay, S. K., Kurata, H. 2012. A review of RFID technology and its managerial applications in different industries. Journal of Engineering and Technology Management, 29 (1), 152167. 